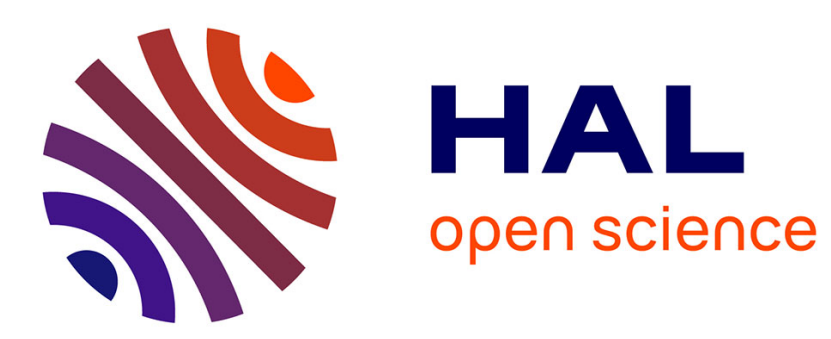

\title{
Stability of marketable payoffs with long-term assets
} Jean-Marc Bonnisseau, Achis Chery

\section{To cite this version:}

Jean-Marc Bonnisseau, Achis Chery. Stability of marketable payoffs with long-term assets. 2013. halshs-00917638

\section{HAL Id: halshs-00917638 \\ https://shs.hal.science/halshs-00917638}

Submitted on 12 Dec 2013

HAL is a multi-disciplinary open access archive for the deposit and dissemination of scientific research documents, whether they are published or not. The documents may come from teaching and research institutions in France or abroad, or from public or private research centers.
L'archive ouverte pluridisciplinaire HAL, est destinée au dépôt et à la diffusion de documents scientifiques de niveau recherche, publiés ou non, émanant des établissements d'enseignement et de recherche français ou étrangers, des laboratoires publics ou privés. 


\section{Documents de Travail du Centre d'Economie de la Sorbonne}

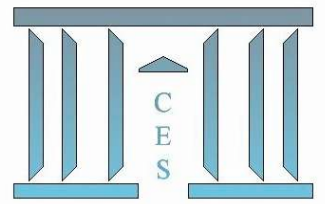

Stability of marketable payoffs with long-term assets

Jean-Marc BonNISSEAU, Achis CHERY

2013.78

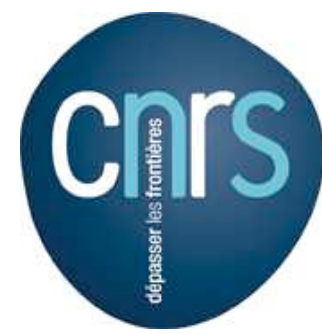




\title{
Stability of marketable payoffs with long-term assets
}

\author{
Jean-Marc Bonnisseau*and Achis Chery ${ }^{\dagger}$
}

September 12, 2013

\begin{abstract}
We consider a stochastic financial exchange economy with a finite dateevent tree representing time and uncertainty and a financial structure with possibly long-term assets. We exhibit a sufficient condition under which the set of marketable payoffs depends continuously on the arbitrage free asset prices. This generalizes previous results of Angeloni-Cornet and Magill-Quinzii involving only short-term assets. We also show that, under the same condition, the useless portfolios do not depend on the arbitrage free asset prices. We then provide an existence result of financial equilibrium for long term nominal assets for any given state prices with assumptions only on the fundamental datas of the economy.
\end{abstract}

Keywords: Incomplete markets, financial equilibrium, multi-period model, long-term assets.

JEL codes: D5, D4, G1

*Paris School of Economics, Université Paris 1 Panthéon Sorbonne, 106-112 Boulevard de l'Hôpital, 75647 Paris Cedex 13, France, Jean-marc.Bonnisseau@univ-paris1.fr

${ }^{\dagger}$ Paris School of Economics, Université Paris 1 Panthéon Sorbonne, 106-112 Boulevard de l'Hôpital, 75647 Paris Cedex 13, France et Centre de Recherche en Gestion et Economie du Développement (CREGED), Université Quisqueya, 218 Haut Turgeau, 6113 Port-au-prince, Haïti, Achis.Chery@malix.univ-paris1.fr 


\section{Contents}

1 Introduction $\quad 3$

2 The $T$-period financial structure $\quad 4$

2.1 Time and uncertainty ................ 5

2.2 The financial structure . . . . . . . . . . . . . 6

$\begin{array}{lll}3 & \text { Stability of the set of marketable payoffs } & 7\end{array}$

3.1 Closedness of the marketable payoff correspondence . . . . . . . 8

3.2 Coincidence of useless portfolios for the payoff and full payoff matrices ..................... . . 10

3.2.1 Statement of Assumption R . . . . . . . . . . 11

3.2.2 Useless portfolios under Assumption R . . . . . . . . . . . 14

4 Existence of equilibrium $\quad 17$

4.1 Financial exchange economy . . . . . . . . . . . . . . . 17

4.2 Financial equilibrium and arbitrage . . . . . . . . . . . . . 18

4.3 The existence result . . . . . . . . . . . . . . . 20

5 Appendix $\quad 23$ 


\section{Introduction}

The literature on the model of stochastic financial exchange economy, also known as the general equilibrium model with incomplete markets, mainly focus on two period economies or, if it consider more than two periods, is restricted to short term assets. Indeed, as soon as we depart from this restrictive frameworks by considering several periods and long term assets, several surprising features happen: the set of arbitrage free asset prices is no more convex and not even a cone (see [4]; the full return matrix may have drop of the rank with respect to the asset prices, whereas it only holds for the spot commodity prices with real assets in two period economies; a useless portfolio for the return matrix may have non zero returns for the full payoff matrix and conversely; the financial markets may be complete or not depending on the arbitrage free asset price. See in $[1,5]$ or below for some simple examples illustrating this phenomena.

These features show that the general framework is much more complex than the two period one and the financial markets may be much more unstable than expected from the analysis of the two period model due to drop of the rank of the full payoff matrix and the switch between complete and incomplete markets with respect to asset prices. From the analytical point of view, a financial equilibrium may not exist under the standard assumptions known as sufficient in the literature.

In this paper, we focus on the stability of the marketable payoffs with respect to the arbitrage free asset prices in presence of long-term assets that is the absence of drops of rank. More precisely, the space of marketable payoffs for a financial structure is the set of payoffs that are reachable by a suitable portfolio through the financial structure that is the payoffs in the image of the full payoff matrix, which is obtained from the payoff matrix by incorporating the opposite of asset prices at the issuance nodes. We provide a sufficient condition on the payoff matrix so that the set of marketable payoffs depends continuously on the asset prices.

We define the stability of the marketable payoffs as the fact that the correspondence which associates the marketable payoffs to the asset prices is lower semi-continuous and has a closed graph. Economically, this means that the super-replication cost is continuous with respect to arbitrage free asset prices or equivalently to state prices.

After introducing notations and the model of a financial structure borrowed from $[1,5]$ in Section 2, we show that the stability of the marketable payoffs holds if the useless portfolios are well defined, that is if they do not depend on the asset price, which emerges on the financial market. A useless portfolio has zero returns at all nodes for the payoff matrix. Actually, we prove that the marketable portfolios are stable when useless portfolios have zero returns for the full payoff matrix whatever is the arbitrage free asset price. In mathematical terms, it means that the kernels of the payoff and the full payoff matrices coincide. But, this property is hardly verifiable since it has to be checked for each arbitrage free asset prices.

That is why we provide a condition, Assumption R, on the payoff matrix in Section 3. It translates the fact that the assets issued at a given node are 
true financial innovations in the sense that their payoffs cannot be replicated by assets issued before. We show that Assumption $\mathbf{R}$ is satisfied if all assets are short term, if there is a unique issuance date, or if there is no overlap of the nodes with non zero returns for two different assets. This last condition is coming from [4] where it is shown that it implies the convexity of the arbitrage free asset price set.

We prove that Assumption $\mathbf{R}$ is a sufficient condition for the stability of marketable payoffs. Actually, we prove that Assumption $\mathbf{R}$ implies the coincidence of the kernels of the payoff and full payoff matrices, that is that the useless portfolios are well defined.

As a byproduct, we remark that we can also characterize a complete financial structure on the payoff matrix that is on the fundamental datas describing the financial structure. Under Assumption R, the financial structure is complete for all arbitrage free asset prices if and only if the rank of the payoff matrix is maximal.

Under Assumption R, we also obtain as corollaries of the above main results the following statements, which are not relevant or absolutely obvious in a two period model but no more always true with long term assets. First, the space of marketable payoffs is the direct sum of the space of marketable payoff space associated to each issuance node. This means that a marketable payoff has a unique decomposition as the sum of marketable payoffs, each of them being associated to a issuance node. A portfolio is useless if and only if each subportfolios obtained by considering only the assets issued at one issuance node is useless. An asset is redundant if and only if it is redundant with the assets issued at the same issuance node. So, this facilitates the identification of such portfolio since one can decompose the problem by considering the payoff sub-matrices associated to the issuance nodes.

In Section 4, we consider a stochastic financial exchange economy with possibly long term nominal assets and restricted participation. We provide an existence result of financial equilibrium for any given state prices when Assumption $\mathbf{R}$ is satisfied by the payoff matrix. These results are based on the existence result (Theorem 3.1) of [1]. Assumption R, which involves only fundamental data of the economy, namely the returns of the assets, allows us to remove an abstract boundedness assumption in [1], which depends on the state price. So our contribution could be seen as the extension of the existence result of [1] to long term assets under Assumption R. It is the first existence result in a multi-period model with long term assets based on assumptions only related to the fundamental datas of the model.

All proofs are gathered in Appendix.

\section{The $T$-period financial structure}

In this section, we present the model and the notations, which are borrowed from Angeloni-Cornet [1] and are essentially the same as those of Magill-Quinzii [5]. 


\subsection{Time and uncertainty}

$\mathrm{We}^{1}$ consider a time structure with $(T+1)$ dates, $t \in \mathcal{T}:=\{0, \ldots, T\}$, and a finite set of agents $\mathcal{I}$. The uncertainty is described by a date-event tree $\mathbb{D}$ of length $T+1$. The set $\mathbb{D}_{t}$ is the set of nodes (also called date-events) that could occur at date $t$ and the family $\left(\mathbb{D}_{t}\right)_{t \in \mathcal{T}}$ defines a partition of the set $\mathbb{D}$; for each $\xi \in \mathbb{D}$, we denote by $t(\xi)$ the unique date $t \in \mathcal{T}$ such that $\xi \in \mathbb{D}_{t}$.

At date $t=0$, there is a unique node $\xi_{0}$, that is $\mathbb{D}_{0}=\left\{\xi_{0}\right\}$. As $\mathbb{D}$ is a tree, each node $\xi$ in $\mathbb{D} \backslash\left\{\xi_{0}\right\}$ has a unique immediate predecessor denoted $\operatorname{pr}(\xi)$ or $\xi^{-}$. The mapping pr maps $\mathbb{D}_{t}$ to $\mathbb{D}_{t-1}$. Each node $\xi \in \mathbb{D} \backslash \mathbb{D}_{T}$ has a nonempty set of immediate successors defined by $\xi^{+}=\left\{\bar{\xi} \in \mathbb{D}: \xi=\bar{\xi}^{-}\right\}$.

For $\tau \in \mathcal{T} \backslash\{0\}$ and $\xi \in \mathbb{D} \backslash \cup_{t=0}^{\tau-1} \mathbb{D}_{t}$, we define $p r^{\tau}(\xi)$ by the recursive formula: $p r^{\tau}(\xi)=p r\left(p r^{\tau-1}(\xi)\right)$. We then define the set of successors and the set of predecessors of $\xi$ as follows:

$$
\begin{aligned}
& \mathbb{D}^{+}(\xi)=\left\{\xi^{\prime} \in \mathbb{D}: \exists \tau \in \mathcal{T} \backslash\{0\} \mid \xi=p r^{\tau}\left(\xi^{\prime}\right)\right\} \\
& \mathbb{D}^{-}(\xi)=\left\{\xi^{\prime} \in \mathbb{D}: \exists \tau \in \mathcal{T} \backslash\{0\} \mid \xi^{\prime}=p r^{\tau}(\xi)\right\}
\end{aligned}
$$

If $\xi^{\prime} \in \mathbb{D}^{+}(\xi)$ [resp. $\xi^{\prime} \in \mathbb{D}^{+}(\xi) \cup\{\xi\}$ ], we shall use the notation $\xi^{\prime}>\xi$ [resp. $\left.\xi^{\prime} \geq \xi\right]$. Note that $\xi^{\prime} \in \mathbb{D}^{+}(\xi)$ if and only if $\xi \in \mathbb{D}^{-}\left(\xi^{\prime}\right)$ and similarly $\xi^{\prime} \in \xi^{+}$if and only if $\xi=\left(\xi^{\prime}\right)^{-}$.

Here, $\mathbb{D}=\left\{\xi_{0}, \xi_{1}, \xi_{2}, \xi_{11}, \xi_{12}, \xi_{13}, \xi_{21}, \xi_{22}\right\}, T=2$, the length of $\mathbb{D}$ is 3 , $\mathbb{D}_{2}=\left\{\xi_{11}, \xi_{12}, \xi_{13}, \xi_{21}, \xi_{22}\right\}, \xi_{1}^{+}=\left\{\xi_{11}, \xi_{12}, \xi_{13}\right\}, \mathbb{D}^{+}\left(\xi_{2}\right)=\left\{\xi_{21}, \xi_{22}\right\}, t\left(\xi_{11}\right)=$ $t\left(\xi_{12}\right)=t\left(\xi_{13}\right)=t\left(\xi_{21}\right)=t\left(\xi_{22}\right)=2, \mathbb{D}^{-}\left(\xi_{11}\right)=\left\{\xi_{0}, \xi_{1}\right\}$.

At each node $\xi \in \mathbb{D}$, there is a spot market on which a finite set $\mathbb{H}=$ $\{1, \ldots, H\}$ of divisible physical goods are exchanged. We assume that each good is perishable, that is, its life does not have more than one date. In this model, a commodity is a pair $(h, \xi)$ of a physical good $h \in \mathbb{H}$ and the node $\xi \in \mathbb{D}$ at which the good is available. Then the commodity space is $\mathbb{R}^{\mathbb{L}}$, where $\mathbb{L}=\mathbb{H} \times \mathbb{D}$. An element $x \in \mathbb{R}^{\mathbb{L}}$ is called a consumption, that is to say $x=(x(\xi))_{\xi \in \mathbb{D}} \in \mathbb{R}^{\mathbb{L}}$, where $x(\xi)=(x(h, \xi))_{h \in \mathbb{H}} \in \mathbb{R}^{\mathbb{H}}$ for each $\xi \in \mathbb{D}$.

We denote by $p=(p(\xi))_{\xi \in \mathbb{D}} \in \mathbb{R}^{\mathbb{L}}$ the vector of spot prices and $p(\xi)=$ $(p(h, \xi))_{h \in \mathbb{H}} \in \mathbb{R}^{\mathbb{H}}$ is called the spot price at node $\xi$. The spot price $p(h, \xi)$ is the price at the node $\xi$ for immediate delivery of one unit of the physical good $h$. Thus the value of a consumption $x(\xi)$ at node $\xi \in \mathbb{D}$ (measured in unit

\footnotetext{
${ }^{1}$ We use the following notations. A $(\mathbb{D} \times \mathcal{J})$-matrix $A$ is an element of $\mathbb{R}^{\mathbb{D} \times \mathcal{J}}$, with entries $\left(a_{\xi}^{j}\right)_{(\xi \in \mathbb{D}, j \in \mathcal{J})}$; we denote by $A_{\xi} \in \mathbb{R}^{\mathcal{J}}$ the $\xi$-th row of $A$ and by $A^{j} \in \mathbb{R}^{\mathbb{D}}$ the $j$-th column of $A$. We recall that the transpose of $A$ is the unique $(\mathcal{J} \times \mathbb{D})$-matrix ${ }^{t} \mathrm{~A}$ satisfying $(A x) \bullet \mathbb{D} y=$ $x \bullet_{\mathcal{J}}\left({ }^{t}\right.$ Ay) for every $x \in \mathbb{R}^{\mathcal{J}}, y \in \mathbb{R}^{\mathbb{D}}$, where $\bullet \mathbb{D}\left[\right.$ resp. $\left.\bullet_{\mathcal{J}}\right]$ denotes the usual inner product in $\mathbb{R}^{\mathbb{D}}\left[\right.$ resp. $\mathbb{R}^{\mathcal{J}}$ ]. We denote by $\operatorname{rank} A$ the rank of the matrix $A$ and by $\operatorname{Vect}(A)$ the range of $A$ that is the linear sub-space spanned by the column vectors of $A$. For every subset $\tilde{\mathbb{D}} \subset \mathbb{D}$ and $\tilde{\mathcal{J}} \subset \mathcal{J}$, the matrix $A_{\tilde{\mathbb{D}}}^{\tilde{\mathcal{J}}}$ is the $(\tilde{\mathbb{D}} \times \tilde{\mathcal{J}})$-sub-matrix of $A$ with entries $a_{\xi}^{j}$ for every $(\xi, j) \in(\tilde{\mathbb{D}} \times \tilde{\mathcal{J}})$. Let $x, y$ be in $\mathbb{R}^{n} ; x \geq y(\operatorname{resp} . x \gg y)$ means $x_{h} \geq y_{h}\left(\right.$ resp. $\left.x_{h}>y_{h}\right)$ for every $h=1, \ldots, n$ and we let $\mathbb{R}_{+}^{n}=\left\{x \in \mathbb{R}^{n}: x \geq 0\right\}, \mathbb{R}_{++}^{n}=\left\{x \in \mathbb{R}^{n}: x \gg 0\right\}$. We also use the notation $x>y$ if $x \geq y$ and $x \neq y$. The Euclidean norm in the different Euclidean spaces is denoted $\|$.$\| and$ the closed ball centered at $x$ and of radius $r>0$ is denoted $\bar{B}(x, r):=\left\{y \in \mathbb{R}^{n} \mid\|y-x\| \leq r\right\}$.
} 


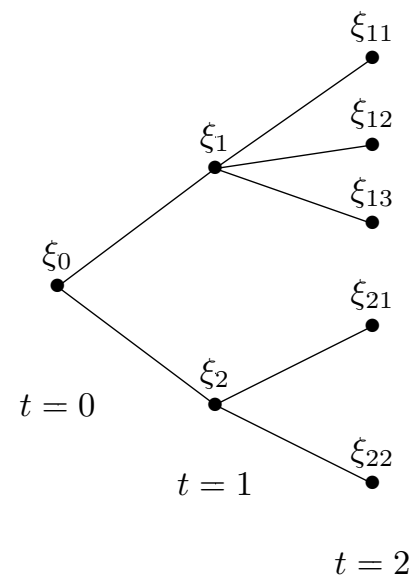

Figure 1: the tree $\mathbb{D}$

account of the node $\xi$ ) is

$$
p(\xi) \bullet \mathbb{H} x(\xi)=\sum_{h \in \mathbb{H}} p(h, \xi) x(h, \xi) .
$$

\subsection{The financial structure}

The financial structure is constituted by a finite set of assets denoted $\mathcal{J}=$ $\{1, \ldots, J\}$. An asset $j \in \mathcal{J}$ is a contract issued at a given and unique node in $\mathbb{D}$ denoted $\xi(j)$, called issuance node of $j$. Each asset is bought or sold only at its issuance node $\xi(j)^{2}$ and yields payoffs only at the successor nodes $\xi^{\prime}$ of $\mathbb{D}^{+}(\xi(j))$. To simplify the notation, we consider the payoff of asset $j$ at every node $\xi \in \mathbb{D}$ and we assume that it is equal to zero if $\xi$ is not a successor of the issuance node $\xi(j)$. The payoff may depend upon the spot price vector $p \in \mathbb{R}^{\mathbb{L}}$ and is denoted by $V_{\xi}^{j}(p)$. Formally, we assume that $V_{\xi}^{j}(p)=0$ if $\xi \notin \mathbb{D}^{+}(\xi(j))$.

A portfolio $z=\left(z^{j}\right)_{j \in \mathcal{J}}$ is an element of $\mathbb{R}^{\mathcal{J}}$. If $z^{j}>0$ [resp. $z^{j}<0$ ], then $\left|z^{j}\right|$ is the quantity of asset $j$ bought [resp. sold] at the issuance node $\xi(j)$.

To summarize a financial structure $\mathcal{F}=\left(\mathcal{J},(\xi(j))_{j \in \mathcal{J}}, V\right)$ consists of

- a set of assets $\mathcal{J}$,

- a node of issuance $\xi(j)$ for each asset $j \in \mathcal{J}$,

- a payoff mapping $V: \mathbb{R}^{\mathbb{L}} \rightarrow \mathbb{R}^{\mathbb{D} \times \mathcal{J}}$ which associates to every spot price $p \in \mathbb{R}^{L}$ the $(\mathbb{D} \times \mathcal{J})$-payoff matrix $V(p)=\left(V_{\xi}^{j}(p)\right)_{\xi \in \mathbb{D}, j \in \mathcal{J}}$ and satisfies the condition $V_{\xi}^{j}(p)=0$ si $\xi \notin \mathbb{D}^{+}(\xi(j))$.

The price of asset $j$ is denoted by $q_{j}$; it is paid at its issuance node $\xi(j)$. We let $q=\left(q_{j}\right)_{j \in \mathcal{J}} \in \mathbb{R}^{\mathcal{J}}$ be the asset price vector.

\footnotetext{
${ }^{2}$ See below Remark 3.2 for a short discussion about the retrading of assets.
} 
The full payoff matrix $W(p, q)$ is the $(\mathbb{D} \times \mathcal{J})$-matrix with the following entries:

$$
W_{\xi}^{j}(p, q):=V_{\xi}^{j}(p)-\delta_{\xi, \xi(j)} q_{j},
$$

where $\delta_{\xi, \xi^{\prime}}=1$ if $\xi=\xi^{\prime}$ and $\delta_{\xi, \xi^{\prime}}=0$ otherwise.

So, given the prices $(p, q)$, the full flow of payoffs for a given portfolio $z \in \mathbb{R}^{\mathcal{J}}$ is $W(p, q) z$ and the full payoff at node $\xi$ is

$$
\begin{aligned}
{[W(p, q) z](\xi) } & :=W_{\xi}(p, q) \bullet \mathcal{J} z=\sum_{j \in \mathcal{J}} V_{\xi}^{j}(p) z^{j}-\sum_{j \in \mathcal{J}} \delta_{\xi, \xi(j)} q_{j} z^{j} \\
& =\sum_{\{j \in \mathcal{J} \mid \xi(j)<\xi\}} V_{\xi}^{j}(p) z^{j}-\sum_{\{j \in \mathcal{J} \mid \xi(j)=\xi\}} q_{j} z^{j}
\end{aligned}
$$

We are now able to define the set of marketable payoffs for $(p, q)$ as:

$$
H(p, q)=\left\{w \in \mathbb{R}^{\mathbb{D}} \mid \exists z \in \mathbb{R}^{\mathcal{J}}, w=W(p, q) z\right\}
$$

which is the range of the matrix $W(p, q)$.

We now recall that for a given spot price $p$, the asset price $q$ is an arbitrage free price if it does not exist a portfolio $z \in \mathbb{R}^{\mathcal{J}}$ such that $W(p, q) z>0$, or, in other words, if $H(p, q) \cap \mathbb{R}_{+}^{\mathbb{D}}=\{0\} . q$ is an arbitrage free price if and only if it exists a so-called state price vector $\lambda \in \mathbb{R}_{++}^{\mathbb{D}}$ such that ${ }^{t} W(p, q) \lambda=0$ (see, e.g. Magill-Quinzii [5]). Taken into account the particular structure of the matrix $W(p, q)$, this is equivalent to

$$
\forall j \in \mathcal{J}, \lambda_{\xi(j)} q_{j}=\sum_{\xi \in \mathbb{D}^{+}(\xi(j))} \lambda_{\xi} V_{\xi}^{j}(p) .
$$

\section{$3 \quad$ Stability of the set of marketable payoffs}

In this section, we study the continuity of the set of marketable payoffs with respect to arbitrage free asset prices for given commodity spot prices. Given a commodity price $p$, we denote by $Q_{p}$ the set of arbitrage free asset prices with respect to $p$. That is,

$$
Q_{p}=\left\{q \in \mathbb{R}^{\mathcal{J}} ; \exists \lambda \in \mathbb{R}_{++}^{\mathbb{D}} \text { satisfying }{ }^{t} W(p, q) \lambda=0\right\}
$$

The continuity of the set of marketable payoffs means that the correspondence $q \rightarrow H(p, q)$ from $Q_{p}$ to $\mathbb{R}^{\mathbb{D}}$ has a closed graph and is lower semicontinuous. In other words, for all sequence $\left(q^{\nu}\right)$ of $Q_{p}$ converging to $q \in Q_{p}$, for all sequence $\left(w^{\nu}\right)$ of $\mathbb{R}^{\mathbb{D}}$ converging to $w$, for all $w^{\prime} \in H(p, q)$, then $w \in H(p, q)$ if $w^{\nu} \in H\left(p, q^{\nu}\right)$ for all $\nu$ and there exists a sequence $\left(w^{\prime \nu}\right)$ of $\mathbb{R}^{\mathbb{D}}$ converging to $w^{\prime}$ and satisfying $w^{\prime \nu} \in H\left(p, q^{\nu}\right)$ for all $\nu$.

In a two period economy, the assets being all issued at date 0 , the structure of $V(p)$ and $W(p, q)$ are very closed since $W(p, q)$ is obtained by filling the first row with the opposite of the asset prices. So, it is quite easy as shown later that the set of marketable payoffs is then continuous. The proposition below states that in a multi-period economy, for each spot price $p$, the correspondence $H(p,$.$) is lower semi-continuous. This is a direct consequence of the fact that$ the matrix $W(p, q)$ depends continuously on $q$.

Proposition 3.1. For each spot price vector $p \in \mathbb{R}^{\mathbb{L}}$, the correspondence $H(p,$. is lower semi-continuous. 


\subsection{Closedness of the marketable payoff correspondence}

The following example shows that the closedness of the graph of the set of marketable payoffs is not granted in a multi-period economy. In all our numerical examples, we assume that there is only one good at each node of the tree and the spot price of the unique good is equal to 1 . Consequently, for the sake of simpler notations, we omit the price $p$ and note the payoff matrix (resp. full payoff matrix) by $V$ (resp. $W(q))$.

Let us consider the financial structure with $\mathbb{D}=\left\{\xi_{0}, \xi_{1}, \xi_{2}, \xi_{21}, \xi_{22}\right\}$ as represented below, $\mathcal{F}=\left(\mathcal{J}, \mathbb{R}^{\mathcal{J}},(\xi(j))_{j \in \mathcal{J}}, V\right)$ such that $\mathcal{J}=\left\{j_{1}, j_{2}, j_{3}, j_{4}\right\}$ and that the first two assets are issued at node $\xi_{0}$, The third asset is issued at node $\xi_{1}$ and the fourth is issued at node $\xi_{2}$.

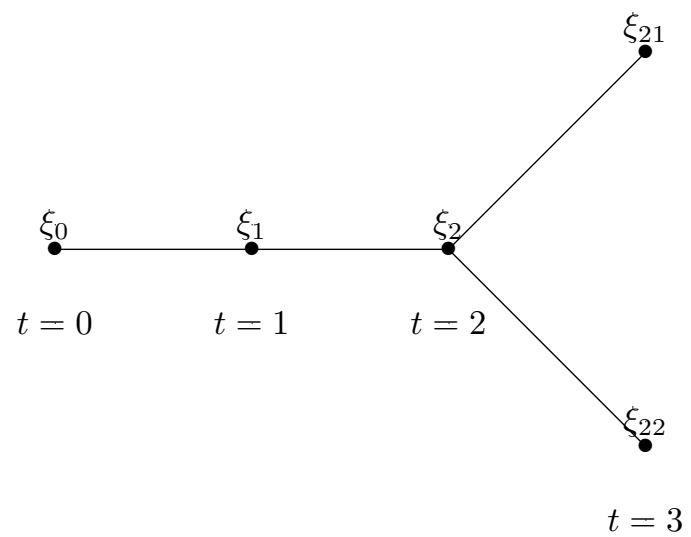

Figure 2: the tree $\mathbb{D}$

The payoff matrix is:

$$
\mathbf{V}=\left[\begin{array}{cccc}
0 & 0 & 0 & 0 \\
0 & 0 & 0 & 0 \\
1 & 0 & 0 & 0 \\
0 & -1 & -1 & 0 \\
-1 & 1 & 1 & 1
\end{array}\right] \begin{aligned}
& \xi_{0} \\
& \xi_{1} \\
& \xi_{2} \\
& \xi_{21} \\
& \xi_{22}
\end{aligned}
$$

We now exhibit a sequence of arbitrage free prices $\left(q^{\nu}\right)$ for which the market is complete but not at the limit $\bar{q}$. Then, we exhibit a payoff $\bar{w}$ such that $\bar{w}$ is marketable for each price $q^{\nu}$ and not at the limit.

Let $\left(q^{\nu}\right)_{\nu \in \mathbb{N}^{*}}=\left(1-\frac{1}{2 \nu}, \frac{1}{\nu}, \frac{1}{\nu}, \frac{1}{2}+\frac{1}{4 \nu}\right)_{\nu \in \mathbb{N}^{*}}$ a sequence of arbitrage free prices associated with the sequence of state prices $\left(\lambda^{\nu}\right)_{\nu \in \mathbb{N}^{*}}=\left(1,1,2,1-\frac{1}{2 \nu}, 1+\right.$ $\left.\frac{1}{2 \nu}\right)_{\nu \in \mathbb{N}^{*}}$. For each $\nu \in \mathbb{N}^{*}$, the full payoff matrix is as follows: 


$$
\mathbf{W}\left(\mathbf{q}^{\nu}\right)=\left[\begin{array}{cccc}
-1+\frac{1}{2 \nu} & -\frac{1}{\nu} & 0 & 0 \\
0 & 0 & -\frac{1}{\nu} & 0 \\
1 & 0 & 0 & -\frac{1}{2}-\frac{1}{4 \nu} \\
0 & -1 & -1 & 0 \\
-1 & 1 & 1 & 1
\end{array}\right] \begin{aligned}
& \xi_{0} \\
& \xi_{1} \\
& \xi_{2} \\
& \xi_{21} \\
& \xi_{22}
\end{aligned}
$$

The market is complete since the rank of the matrix $W\left(q^{\nu}\right)$ is 4 . Indeed, the determinant of the square sub-matrix of size 4 constituted with the rows $\xi_{1}, \xi_{2}, \xi_{21}$ and $\xi_{22}$ and the columns of $W\left(q^{\nu}\right)$ is equal to $-\frac{2 \nu+1}{4 \nu^{2}} \neq 0$.

At the limit, $\bar{q}=\left(1,0,0, \frac{1}{2}\right) . \bar{q}$ is an arbitrage free price associated to $\lambda=$ $(1,1,2,1,1)$. We have

$$
\mathbf{W}(\overline{\mathbf{q}})=\left[\begin{array}{cccc}
\mathbf{- 1} & \mathbf{0} & 0 & 0 \\
0 & 0 & \mathbf{0} & 0 \\
1 & 0 & 0 & \mathbf{- 1 / 2} \\
0 & -1 & -1 & 0 \\
-1 & 1 & 1 & 1
\end{array}\right] \begin{gathered}
\xi_{0} \\
\xi_{1} \\
\xi_{2} \\
\xi_{21} \\
\xi_{22}
\end{gathered}
$$

Here the rank of the matrix $W(\bar{q})$ is 3 because it has 2 identical columns and the square sub-matrix of size 3 formed with the rows $\xi_{0}, \xi_{21}, \xi_{22}$ and columns 1 , 3 and 4 has a nonzero determinant. So the market is incomplete at $\bar{q}$.

This drop of the rank of the full payoff matrix leads to the inability to replicate some payoff at the limit. Indeed, let $w=\left(0,-1, \frac{1}{2}, 0,0\right)$. For all $\nu$, $w=W\left(q^{\nu}\right) z^{\nu}$ with $z^{\nu}=\left(\frac{2 \nu}{2 \nu-1},-\nu, \nu, \frac{2 \nu}{2 \nu-1}\right)$, hence $w$ is marketable for $q^{\nu}$ but not for $\bar{q}$. Indeed, suppose that there exists $z \in \mathbb{R}^{4}$ such that $w=W(\bar{q}) z$. Then,

$$
\left(0,-1, \frac{1}{2}, 0,0\right)=\left(-z_{1}, 0, z_{1}-\frac{z_{4}}{2},-z_{2}-z_{3},-z_{1}+z_{2}+z_{3}+z_{4}\right) \Rightarrow 0=-1
$$

a contradiction.

We now provide a sufficient general condition under which the marketable payoff correspondence has a closed graph. This condition is based on the useless portfolios for a financial structure, that is a portfolio with zero returns at all nodes. Formally, for a spot price vector $p \in \mathbb{R}^{\mathbb{L}}$, a portfolio $z \in \mathbb{R}^{\mathcal{J}}$ is useless if $V(p) z=0$, that is $z$ belongs to the kernel of $V(p)$.

Proposition 3.2. Let $p \in \mathbb{R}^{\mathbb{L}}$ be a given spot price vector. If for all arbitrage free asset price $q \in Q_{p}$ the kernel of $W(p, q)$ is equal to the kernel of $V(p)$ then the marketable payoff correspondence defined by

$$
q \rightarrow H(p, q)=\left\{w \in \mathbb{R}^{\mathbb{D}} \mid \exists z \in \mathbb{R}^{\mathcal{J}}, w=W(p, q) z\right\}
$$

has a closed graph.

The condition on the kernel of $V(p)$ and $W(p, q)$ means that $z$ is a useless portfolio if and only if its returns are all equal to 0 for the full payoff matrix whatever is the arbitrage free asset price. Then, the definition of useless portfolios is not ambiguous since it does not depend on asset prices and a useless portfolio has zero returns for the payoff matrix as well as for the full payoff 
matrix. So the stability of the marketable payoffs is a consequence of the fact that we can identify the useless portfolio either on the payoff matrix or on the full payoff matrix whatever is the arbitrage free asset price.

The equality of the kernels is obviously satisfied in a two period financial structure for a arbitrage free asset price but this is no longer true with longterm assets. Indeed, if we consider the same example as above and if we let $q^{1}=(0,0,0,1) \cdot q$ is an arbitrage free price because ${ }^{t} W\left(q^{1}\right) \lambda^{1}=0$ with $\lambda^{1}=$ $(1,1,1,1,1) \in \mathbb{R}_{++}^{5}$. The full payoff matrix associated with this price $q^{1}$ is the following:

$$
\mathbf{W}\left(\mathbf{q}^{\mathbf{1}}\right)=\left[\begin{array}{cccc}
\mathbf{0} & \mathbf{0} & 0 & 0 \\
0 & 0 & \mathbf{0} & 0 \\
1 & 0 & 0 & \mathbf{- 1} \\
0 & -1 & -1 & 0 \\
-1 & 1 & 1 & 1
\end{array}\right] \begin{gathered}
\xi_{0} \\
\xi_{1} \\
\xi_{2} \\
\xi_{21} \\
\xi_{22}
\end{gathered}
$$

The rank of $W\left(q^{1}\right)$ is 2 since its kernel is of dimension 2 and equal to

$$
\left\{z \in \mathbb{R}^{4} \mid z_{1}=z_{4}, z_{2}+z_{3}=0\right\}
$$

and it is not included in the kernel of $V$, which is equal to

$$
\left\{z \in \mathbb{R}^{4} \mid z_{1}=z_{4}=0, z_{2}+z_{3}=0\right\}
$$

So the portfolio $z=(1,1,-1,1)$ has zero returns at all nodes for the full payoff matrix but is not useless since its return is $(0,0,1,0,0)$ for the payoff matrix.

Now, consider a second asset price $q^{2}=(-1,1,1,2) . q^{2}$ is an arbitrage free price because ${ }^{t} W\left(q^{2}\right) \lambda^{2}=0$ with $\lambda^{2}=(1,1,1,1,2) \in \mathbb{R}_{++}^{5}$. The full payoff matrix associated with this price $q^{2}$ is the following:

$$
\mathbf{W}\left(\mathbf{q}^{2}\right)=\left[\begin{array}{cccc}
\mathbf{1} & \mathbf{- 1} & 0 & 0 \\
0 & 0 & \mathbf{- 1} & 0 \\
1 & 0 & 0 & \mathbf{- 2} \\
0 & -1 & -1 & 0 \\
-1 & 1 & 1 & 1
\end{array}\right] \begin{gathered}
\xi_{0} \\
\xi_{1} \\
\xi_{2} \\
\xi_{21} \\
\xi_{22}
\end{gathered}
$$

The rank of $W\left(q^{2}\right)$ is 4 because its kernel is reduced to $\{0\}$. Hence the kernel of $W\left(q^{2}\right)$ is included and not equal to the kernel of $V$. The portfolio $z^{\prime}=$ $(0,1,-1,0)$ is useless but its returns for the full payoff matrix are $(-1,1,0,0,0)$.

\subsection{Coincidence of useless portfolios for the payoff and full payoff matrices}

In Proposition 3.2, the stability of marketable payoffs is based on the equality of the kernels of payoff matrices, which should be checked for each arbitrage free asset price. This kind of assumption exhibits the drawback that it involves an endogenous variable, the asset price, which is determined by the market mechanism. The purpose of this section is to provide a sufficient condition (Assumption $\mathbf{R}$ ) on the payoff matrix $V(p)$ to get the equality of the kernel for 
each arbitrage free price in presence of long-term assets or, in financial words, a definition of useless portfolios independent from the asset prices. This generalizes the two-period and the short term asset cases. Hence, the stability of the marketable payoffs can be determined on the fundamentals of the financial structure.

Furthermore, Assumption $\mathbf{R}$ has several other interesting consequences. It allows to check the completeness of the asset structure on $V(p)$ for all arbitrage free asset prices. Indeed, in the example given above, we remark that the market is complete in the sense that $W\left(q^{2}\right)$ has the maximal rank 4 for the arbitrage free price $q^{2}$, but the market is incomplete for the arbitrage free price $q^{1}$ since the rank of $W\left(q^{1}\right)$ is only 2 . So, even if $V$ has a rank equal to 3 , the market is complete for some asset prices like $q^{2}$ and "more" incomplete than $V$ for some other asset prices like $q^{1}$. We state below a proposition showing that, under Assumption $\mathbf{R}$, if $V(p)$ has a maximal rank, then the markets are complete for all arbitrage free asset prices.

Under Assumption $\mathbf{R}$, we also prove that the marketable payoff set can be decomposed as the direct sum of the marketable payoff sets generated at each issuance node. So each marketable payoff has a unique decomposition as the sum of marketable payoffs generated by the assets issued at the same node. Given a marketable payoff, this simplifies a lot the computation of the portfolio, which replicates it. This also helps for the identification of redundant assets, since this can be done issuance node by issuance node so by considering only a much smaller set of assets.

We now introduce some additional notations. For all $\xi \in \mathbb{D} \backslash \mathbb{D}_{T}, \mathcal{J}(\xi)$ is the set of assets issued at the node $\xi$, that is $\mathcal{J}(\xi)=\{j \in \mathcal{J} \mid \xi(j)=\xi\}$ and $n(\xi)$ is the cardinal of $\mathcal{J}(\xi) \cdot \mathcal{J}\left(\mathbb{D}^{-}(\xi)\right)$ is the set of assets issued at a predecessor of $\xi$, that is $\mathcal{J}\left(\mathbb{D}^{-}(\xi)\right)=\{j \in \mathcal{J} \mid \xi(j)<\xi\}$. For all $t \in\{0, \ldots, T-1\}$, we denote by $\mathcal{J}_{t}$ the set of assets issued at date $t$, that is, $\mathcal{J}_{t}=\left\{j \in \mathcal{J} \mid \xi(j) \in \mathbb{D}_{t}\right\}$.

Let $\left(\tau_{1}, \ldots, \tau_{k}\right)$ such that $0 \leq \tau_{1}<\tau_{2}<\ldots<\tau_{k} \leq T-1$ be the dates at which there is at least the issuance of one asset, that is $\mathcal{J}_{\tau_{\kappa}} \neq \emptyset$. For $\kappa=1, \ldots, k$, let $\mathbb{D}_{\tau_{\kappa}}^{e}$ be the set of nodes at date $\tau_{\kappa}$ at which there is the issuance of at least one asset. $\mathbb{D}^{e}=\cup_{\kappa=1}^{k} \mathbb{D}_{\tau_{\kappa}}^{e}$ is the set of nodes at which there is the issuance of at least one asset. We remark that

$$
\bigcup_{\tau \in\{0, \ldots, T-1\}} \mathcal{J}_{\tau}=\bigcup_{\kappa \in\{1, \ldots, k\}} \mathcal{J}_{\tau_{\kappa}}=\mathcal{J}, \quad J=\sum_{\kappa \in\{1, \ldots, k\}} \# \mathcal{J}_{\tau_{\kappa}}
$$

and for all $\tau \in\left\{\tau_{1}, \ldots, \tau_{k}\right\}, \bigcup_{\xi \in \mathbb{D}_{\tau}} \mathcal{J}(\xi)=\mathcal{J}_{\tau}$.

\subsubsection{Statement of Assumption R}

Let $p \in \mathbb{R}^{\mathbb{L}}$ be a given spot price vector. We now state our sufficient condition on the matrix $V(p)$.

Assumption R. $\forall \xi \in \mathbb{D}^{e}$,

$$
\operatorname{Vect}\left(V_{\mathbb{D}^{+}(\xi)}^{\mathcal{J}\left(\mathbb{D}^{-}(\xi)\right)}(p)\right) \bigcap \operatorname{Vect}\left(V_{\mathbb{D}^{+}(\xi)}^{\mathcal{J}(\xi)}(p)\right)=\{0\} .
$$


This assumption means that the returns of the assets issued at a node $\xi$ are not redundant with the returns of the assets issued at a predecessor node of $\xi$. So, the issuance of additional assets at $\xi$ leads to a true financial innovation since the payoffs in the successors of $\xi$ cannot be replicated by the payoffs of a portfolio built with the assets issued before $\xi$.

In the following lemma, we show that if Assumption $\mathbf{R}$ holds true for the financial structure $\mathcal{F}$, it is also true for any financial substructure $\mathcal{F}^{\prime}$ of $\mathcal{F}$ obtained by considering only a subset $\mathcal{J}^{\prime}$ of the set of assets $\mathcal{J}$.

Lemma 3.1. Let

$$
\mathcal{F}=\left(\mathcal{J},(\xi(j))_{j \in \mathcal{J}}, V\right) \quad \mathcal{F}^{\prime}=\left(\mathcal{J}^{\prime},(\xi(j))_{j \in \mathcal{J}^{\prime}}, V^{\prime}\right)
$$

two financial structures such as $\mathcal{J}^{\prime} \subset \mathcal{J}$ and $V^{\prime}=V^{\mathcal{J}^{\prime}}$. If Assumption $\boldsymbol{R}$ holds true for the structure $\mathcal{F}$ then it holds also true for the structure $\mathcal{F}^{\prime}$.

Remark 3.1. The converse of Lemma 3.1 is not true. Let us consider an economy with three periods such as: $\mathbb{D}=\left\{\xi_{0}, \xi_{1}, \xi_{2}, \xi_{11}, \xi_{12}, \xi_{21}, \xi_{22}\right\}$.

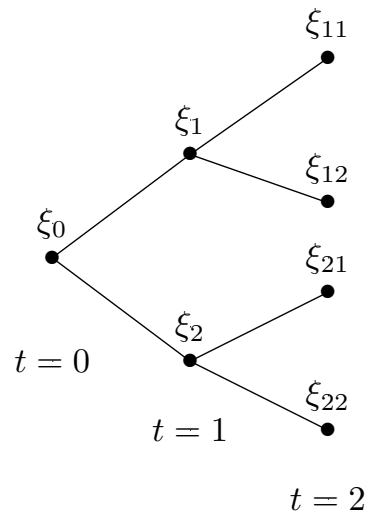

Figure 3: the tree $\mathbb{D}$

There are three assets issued at nodes $\xi_{0}, \xi_{1}$ and $\xi_{2}$. The return matrix is $V$ and the one of the substructure where we keep only the two first assets is $V^{\prime}$ :

$$
V=\left[\begin{array}{lll}
0 & 0 & 0 \\
0 & 0 & 0 \\
0 & 0 & 0 \\
0 & 0 & 0 \\
0 & 1 & 0 \\
1 & 0 & 1 \\
0 & 0 & 0
\end{array}\right] \begin{aligned}
& \xi_{0} \\
& \xi_{1} \\
& \xi_{2} \\
& \xi_{11} \\
& \xi_{12} \\
& \xi_{21} \\
& \xi_{22}
\end{aligned} \quad V^{\prime}=\left[\begin{array}{ll}
0 & 0 \\
0 & 0 \\
0 & 0 \\
0 & 0 \\
0 & 1 \\
1 & 0 \\
0 & 0
\end{array}\right] \begin{aligned}
& \xi_{0} \\
& \xi_{1} \\
& \xi_{2} \\
& \xi_{11} \\
& \xi_{12} \\
& \xi_{21} \\
& \xi_{22}
\end{aligned}
$$

One remark that Assumption $\mathbf{R}$ is not satisfied for $V$ for the node $\xi_{2}$ whereas it holds true for the reduced financial structure.

The next proposition provides some sufficient conditions under which Assumption $\mathbf{R}$ holds true. 
Proposition 3.3. Given a spot price vector $p \in \mathbb{R}^{\mathbb{L}}$. The return matrix $V(p)$ satisfies Assumption $\mathbf{R}$ if one of the following condition is satisfied:

(i) For all $j \in \mathcal{J}$, asset $j$ is a short term asset in the sense that $V_{\xi^{\prime}}^{j}(p)=0$ if $\xi^{\prime} \notin \xi^{+}(j)$.

(ii) All assets are issued at the same date $\tau_{1}$.

(iii) For all $\xi \in \mathbb{D}^{e}, \mathbb{D}^{+}(\xi) \cap \mathbb{D}^{e}=\emptyset$, which means that if an asset is issued at a node $\xi$, then no assets is issued at a successor of $\xi$.

(iv) For all $\xi \in \mathbb{D}$ and for all $j, \ell \in \mathcal{J}, V_{\xi}^{j}(p) V_{\xi}^{l}(p)=0$ if $\xi(j) \neq \xi(l)$.

(v) For all $\left(\xi, \xi^{\prime}\right) \in\left(\mathbb{D}^{e}\right)^{2}$, if $\xi<\xi^{\prime}$, then $V_{\mathbb{D}^{+}\left(\xi^{\prime}\right)}^{\mathcal{J}(\xi)}=0$, which means that if an asset $j$ is issued at node $\xi$ and another one at a successor $\xi^{\prime}$, then the return of $j$ at the successors of $\xi^{\prime}$ are equal to 0 .

The proof of this proposition is left to the reader. It is a consequence of the fact that either $\mathcal{J}\left(\mathbb{D}^{-}(\xi)\right)$ is an empty set or $\operatorname{Vect}\left(V_{\mathbb{D}^{+}(\xi)}^{\mathcal{J}\left(\mathbb{D}^{-}(\xi)\right)}(p)\right)=\{0\}$. Note that Condition (iv) is introduced in [4] to study the structure of the arbitrage free asset prices.

Note that in a two period model, there are only short term assets, so all financial structures satisfy Assumption $\mathbf{R}$ in that case.

If the assets issued at each node are linearly independent, then Assumption $\mathbf{R}$ is derived from a slightly weaker assumption where we only deal with the returns at the immediate successors of a node $\xi$ instead of looking at the whole returns for all successors.

Corollary 3.1. Given a spot price vector $p \in \mathbb{R}^{\mathbb{L}}$. Let us assume that:

1) $\forall \xi \in \mathbb{D}^{e}, \operatorname{rank} V(p)_{\xi^{+}}^{\mathcal{J}(\xi)}(p)=n(\xi)$

and

2) $\operatorname{Vect}\left(V_{\xi^{+}}^{\mathcal{J}\left(\mathbb{D}^{-}(\xi)\right)}(p)\right) \cap \operatorname{Vect}\left(V_{\xi^{+}}^{\mathcal{J}(\xi)}(p)\right)=\{0\}$.

Then Assumption $\mathbf{R}$ is satisfied.

Remark 3.2. In Magill and Quinzii [5], it is assumed that a long-term asset is re-traded at each nodes after its issuance node. In Angeloni and Cornet [1], it is shown that a financial structure with re-trading is equivalent to a financial structure without re-trading by considering that a re-trade is equivalent to the issuance of a new asset.

We remark that if the financial structure has long-term assets with retrading, then Assumption $\mathbf{R}$ may hold true without re-trading but not with re-trading. Let us give an example. Let us consider the date-event tree $\mathbb{D}$ as above in Remark 3.1. Two assets are issued at $\xi_{0}$ with dividend processes

$$
V^{1}=(0,(0,0),(1,0,1,0)) \quad V^{2}=(0,(0,0),(0,1,0,1))
$$

so Assumption $\mathbf{R}$ is satisfied thanks to Proposition 3.3 (ii). 
If these two assets are re-traded at each non-terminal successor node of $\xi_{0}$, for all arbitrage free price $q=\left(q_{1}\left(\xi_{0}\right), q_{2}\left(\xi_{0}\right), q_{1}\left(\xi_{1}\right), q_{2}\left(\xi_{1}\right), q_{1}\left(\xi_{2}\right), q_{2}\left(\xi_{2}\right)\right)$, the full payoff matrix $\mathbf{W}_{\mathbf{M Q}}(\mathbf{q})$ is:

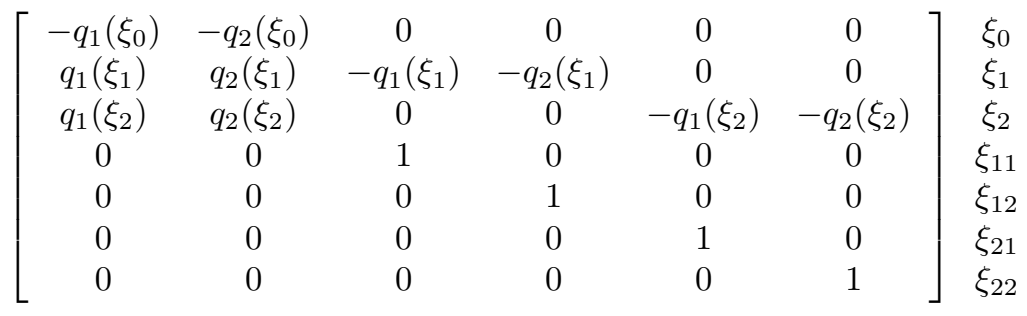

But if, following the methodology of Angeloni-Cornet [1], we consider an equivalent financial structure with 6 assets without re-trading, we obtain the following full payoff matrix with $\tilde{q}=\left(\tilde{q}_{1}, \tilde{q}_{2}, \tilde{q}_{11}, \tilde{q}_{12}, \tilde{q}_{21}, \tilde{q}_{22}\right)$,

$$
\mathbf{W}_{\mathbf{A C}}(\tilde{\mathbf{q}})=\left[\begin{array}{cccccc}
-\tilde{q}_{1} & -\tilde{q}_{2} & 0 & 0 & 0 & 0 \\
0 & 0 & -\tilde{q}_{11} & -\tilde{q}_{12} & 0 & 0 \\
0 & 0 & 0 & 0 & -\tilde{q}_{21} & -\tilde{q}_{22} \\
1 & 0 & 1 & 0 & 0 & 0 \\
0 & 1 & 0 & 1 & 0 & 0 \\
1 & 0 & 0 & 0 & 1 & 0 \\
0 & 1 & 0 & 0 & 0 & 1
\end{array}\right] \begin{gathered}
\xi_{0} \\
\xi_{1} \\
\xi_{2} \\
\xi_{11} \\
\xi_{12} \\
\xi_{21} \\
\xi_{22}
\end{gathered}
$$

We remark that the two financial structures are equivalent when $q=\tilde{q}$ since, by performing elementary operations on the columns of $W_{A C}(q)$, we obtain $W_{M Q}(q)$. Assumption $\mathbf{R}$ is not satisfied because the returns of assets issued at nodes $\xi_{1}$ and $\xi_{2}$ are redundant with the return of assets issued at node $\xi_{0}$. As already remarked in Magill-Quinzii [5], the rank of the full payoff matrix $W_{M Q}(q)$, so the kernel, depends on the asset price vector $q$.

\subsubsection{Useless portfolios under Assumption R}

Now, we state the main result of this section, which actually is the justification of Assumption R.

Proposition 3.4. Given a spot price vector $p \in \mathbb{R}^{\mathbb{L}}$. If the return matrix $V(p)$ satisfies Assumption $\mathbf{R}$, then for all arbitrage free price $q, \operatorname{Ker} V(p)=$ $\operatorname{Ker} W(p, q)$.

This proposition means that the sets of useless portfolios for the payoff matrix and the full payoff matrix always coincide whatever is the arbitrage free asset price. Together with Proposition 3.2, we then deduces from Proposition 3.4 that under Assumption $\mathbf{R}$, the marketable payoffs are stable.

Remark 3.3. Assumption $\mathbf{R}$ is sufficient but not necessary. Indeed, for the following financial structure, Assumption $\mathbf{R}$ does not hold true and yet, for any (arbitrage free or not) price of assets $q, \operatorname{Ker} V(p)=\operatorname{Ker} W(p, q)$. Let us consider the date-event tree $\mathbb{D}$ as above in Remark 3.1. Three assets are issued, two at 
$\xi_{0}$ and one at $\xi_{1}$. For all asset price $q=\left(q_{1}, q_{2}, q_{3}\right)$, the return matrix and the full return matrix are:

$$
\mathbf{V}=\left[\begin{array}{lll}
0 & 0 & 0 \\
1 & 0 & 0 \\
0 & 1 & 0 \\
1 & 0 & 1 \\
0 & 1 & 1 \\
0 & 0 & 0 \\
0 & 0 & 0
\end{array}\right] \begin{aligned}
& \xi_{0} \\
& \xi_{2} \\
& \xi_{11} \\
& \xi_{12} \\
& \xi_{21} \\
& \xi_{22}
\end{aligned} \text { and } \quad \mathbf{W}(\mathbf{q})=\left[\begin{array}{ccc}
-q_{1} & -q_{2} & 0 \\
1 & 0 & -q_{3} \\
0 & 1 & 0 \\
1 & 0 & 1 \\
0 & 1 & 1 \\
0 & 0 & 0 \\
0 & 0 & 0
\end{array}\right] \begin{aligned}
& \xi_{0} \\
& \xi_{1} \\
& \xi_{2} \\
& \xi_{11} \\
& \xi_{12} \\
& \xi_{21} \\
& \xi_{22}
\end{aligned}
$$

One easily checks $\operatorname{rank} V=\operatorname{rank} W(q)=3$ so $\operatorname{Ker} V=\{0\}=\operatorname{Ker} W(q)$ whatever is the asset price $q$.

The proof of Proposition 3.4 uses as a key step the following proposition on the equality of the rank of the payoff matrices and on the decomposition of the marketable payoff space.

Proposition 3.5. Given a spot price vector $p \in \mathbb{R}^{\mathbb{L}}$. If the return matrix $V(p)$ satisfies Assumption $\mathbf{R}$, then for all arbitrage free price $q, \operatorname{rank} V(p)=$ $\operatorname{rank} W(p, q)$.

Furthermore, for every arbitrage free price $q$, the linear subspaces $\left\{\operatorname{Im} V^{\mathcal{J}(\xi)}(p)\right\}_{\xi \in \mathbb{D}^{e}}$ and $\left\{\operatorname{Im} W^{\mathcal{J}(\xi)}(p, q)\right\}_{\xi \in \mathbb{D}^{e}}$ are linearly independent ${ }^{3}$ so $\operatorname{Im} V(p)=\bigoplus_{\xi \in \mathbb{D}^{e}} \operatorname{Im} V^{\mathcal{J}(\xi)}(p)$ and $\operatorname{Im} W(p, q)=\bigoplus_{\xi \in \mathbb{D}^{e}} \operatorname{Im} W^{\mathcal{J}(\xi)}(p, q)$.

Condition (i) of Proposition 3.3 shows that Proposition 3.5 is a generalization of Proposition 5.2. b) and c) in Angeloni-Cornet [1] and of Magill-Quinzii [5] where only short-term assets are considered.

The following corollary is deduced from Proposition 3.5. It means that under Assumption $\mathbf{R}$, the structure has no redundant asset if and only if it is the case at each issuance node.

Corollary 3.2. Let $p \in \mathbb{R}^{\mathbb{L}}$ be a spot price. Let $\mathcal{F}=\left\{\mathcal{J}, \mathbb{R}^{\mathcal{J}},(\xi(j))_{j \in \mathcal{J}}, V\right\}$ be a financial structure satisfying Assumption $\boldsymbol{R}$ at the spot price $p$. Then

(A) the two following conditions are equivalent:

(i) for every $\xi \in \mathbb{D}^{e}$, the family $\left\{V^{j}(p)\right\}_{j \in \mathcal{J}(\xi)}$ is linearly independent;

(ii) the family $\left\{V^{j}(p)\right\}_{j \in \mathcal{J}}$ is linearly independent.

(B) for every arbitrage free price $q \in \mathbb{R}^{\mathcal{J}}$, the two following conditions are equivalent:

(i) for every $\xi \in \mathbb{D}^{e}$, the family $\left\{W^{j}(p, q)\right\}_{j \in \mathcal{J}(\xi)}$ is linearly independent;

\footnotetext{
${ }^{3}$ Linear subspaces $U_{1}, U_{2}, \ldots, U_{m}$ (with $U_{\ell} \neq\{0\}$ for all $\ell$ ) are linearly independent if for all $\left(u_{\ell}\right) \in \prod_{\ell=1}^{m} U_{\ell}, u_{1}+u_{2}+\ldots+u_{m}=0$ implies $u_{\ell}=0$ for all $\ell$. The sum of the linearly independent linear subspaces is called a direct sum of these linear subspaces and is denoted by $\bigoplus_{\ell=1}^{m} U_{\ell}=U_{1} \oplus \ldots \oplus U_{m}$. Note that for all $u \in \bigoplus_{\ell=1}^{m} U_{\ell}$, there exists a unique $\left(u_{\ell}\right) \in \prod_{\ell=1}^{m} U_{\ell}$ such that $u=\sum_{\ell=1}^{m} u_{\ell}$.
} 
(ii) the family $\left\{W^{j}(p, q)\right\}_{j \in \mathcal{J}}$ is linearly independent.

From Proposition 3.5, we deduce that an asset $j$ is redundant ${ }^{4}$ if and only if it is linearly dependent with other assets issued at the same node as it i.e., at the node $\xi(j)$. So the identification of redundant assets is much easier since it suffices to consider for each node $\xi \in \mathbb{D}^{e}$, the sub-matrix $V^{\mathcal{J}(\xi)}(p)$ consisting of the columns corresponding to the payoffs of assets issued at the node $\xi$.

Corollary 3.3. Let $p \in \mathbb{R}^{\mathbb{L}}$ be a spot price given, let $\mathcal{F}=\left\{\mathcal{J}, \mathbb{R}^{\mathcal{J}},(\xi(j))_{j \in \mathcal{J}}, V\right\}$ be a financial structure satisfying Assumption $\boldsymbol{R}$ at the spot price $p$. Then the two following conditions are equivalent:

(i) the asset $j_{0}$ is redundant at the spot price $p$;

(ii) there exists a family $\left\{\alpha_{j}\right\}_{j \in \mathcal{J}\left(\xi\left(j_{0}\right)\right) \backslash\left\{j_{0}\right\}} \in \mathbb{R}^{\mathcal{J}\left(\xi\left(j_{0}\right)\right) \backslash\left\{j_{0}\right\}}$ such that $V^{j_{0}}(p)=\sum_{j \in \mathcal{J}\left(\xi\left(j_{0}\right)\right) \backslash\left\{j_{0}\right\}} \alpha_{j} V^{j}(p)$.

Remark 3.4. Without Assumption $\mathbf{R}$, the previous results may be false. Indeed, let $\mathcal{F}=\left\{\mathcal{J}, \mathbb{R}^{\mathcal{J}},(\xi(j))_{j \in \mathcal{J}}, V\right\}$ be a financial structure with nominal assets such that $\mathbb{D}=\left\{\xi_{0}, \xi_{1}, \xi_{2}, \xi_{3}, \xi_{4}\right\}, \mathcal{J}=\left\{j^{1}, j^{2}, j^{3}\right\}$. The first asset is issued at node $\xi_{0}$, the second and the third assets are issued at node $\xi_{1}$.

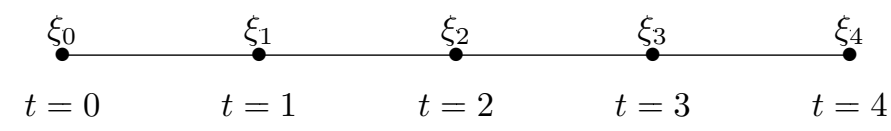

Figure 4: the tree $\mathbb{D}$

The payoff matrice is:

$$
V=\left(\begin{array}{lll}
0 & 0 & 0 \\
0 & 0 & 0 \\
1 & 1 & 0 \\
1 & 0 & 1 \\
2 & 1 & 1
\end{array}\right) \begin{aligned}
& \xi_{0} \\
& \xi_{1} \\
& \xi_{2} \\
& \xi_{3} \\
& \xi_{4}
\end{aligned}
$$

Clearly, $\mathcal{F}$ does not satisfy Assumption $\mathbf{R}$, the family $\left\{\operatorname{Im} V^{\mathcal{J}(\xi)}\right\}_{\xi \in \mathbb{D}^{e}}$ is linearly dependent, for every $\xi \in \mathbb{D}^{e}$, the family $\left\{V^{j}\right\}_{j \in \mathcal{J}(\xi)}$ is linearly independent while the family $\left\{V^{j}\right\}_{j \in \mathcal{J}}$ is linearly dependent.

Remark 3.5. If the price $q$ exhibits an arbitrage, then even under Assumption $\mathbf{R}$, the rank of $V(p)$ and the rank of $W(p, q)$ may be different. With a three dates economy where $\mathbb{D}=\left\{\xi_{0}, \xi_{1}, \xi_{2}, \xi_{11}, \xi_{12}, \xi_{21}, \xi_{22}\right\}$ as above in Remark 3.1,

\footnotetext{
${ }^{4}$ Recall that an asset $j_{0}$ of a redundant for the payoff matrix $V$ at the spot price $p$ if the column vector $V^{j_{0}}(p)$ representing its payoffs on $\mathbb{D}$ is a linear combination of the column vectors representing the payoffs of other assets, i.e., if there exists $\alpha=\left(\alpha^{j}\right)_{j \in \mathcal{J} \backslash\left\{j_{0}\right\}} \in \mathbb{R}^{\mathcal{J} \backslash\left\{j_{0}\right\}}$ such that $V^{j_{0}}(p)=\sum_{j \in \mathcal{J} \backslash\left\{j_{0}\right\}} \alpha^{j} V^{j}(p)$.
} 
two assets issued at $\xi_{0}$ and one asset issued at $\xi_{1}$, the asset price $q=\left(1, \frac{3}{2}, 1\right)$, then

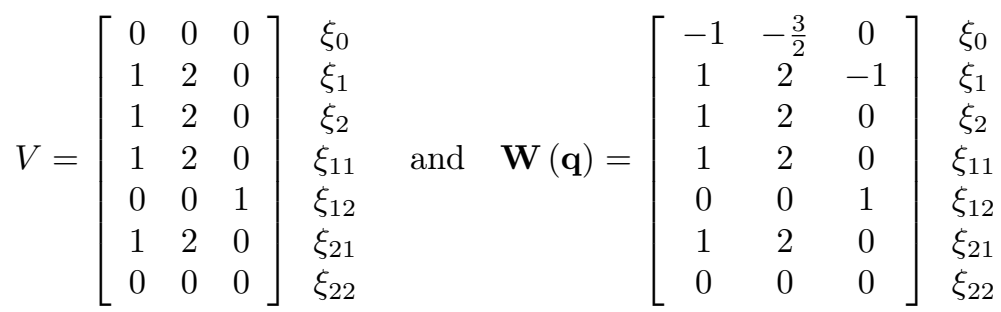

We note that $\operatorname{rank} V=2<\operatorname{rank} W(q)=3$. Nevertheless, the following result shows that if the payoff vectors are not redundant at each node, then the equality of ranks holds true even with an arbitrage price.

Proposition 3.6. Given a spot price vector $p \in \mathbb{R}^{\mathbb{L}}$. Let us assume that $V$ satisfies Assumption R.

1) For all price $q \in \mathbb{R}^{\mathcal{J}}, \operatorname{rank} V(p) \leq \operatorname{rank} W(p, q)$.

2) Furthermore, if for all $\xi \in \mathbb{D}^{e}, \operatorname{rank} V^{\mathcal{J}(\xi)}(p)=n(\xi)$, the number of assets issued at this node, then $\operatorname{rank} V(p)=\operatorname{rank} W(p, q)$ for all price $q \in \mathbb{R}^{\mathcal{J}}$.

The following corollary is a generalization of Proposition 3.5, which could be interesting in presence of market restrictions.

Corollary 3.4. Given a spot price vector $p \in \mathbb{R}^{\mathbb{L}}$. Let $\mathcal{F}=\left(\mathcal{J},(\xi(j))_{j \in \mathcal{J}}, V\right)$ be a financial structure such that Assumption $\mathbf{R}$ is satisfied and let $G$ be a linear subspace of $\mathbb{R}^{\mathcal{J}}$. Then for all arbitrage free price $q$, $\operatorname{dim}[W(p, q) G]=$ $\operatorname{dim}[V(p) G]$.

Proposition 3.5 is merely the case where $G=\mathbb{R}^{\mathcal{J}}$. The proof of Corollary 3.4 is deduced from Proposition 3.4 and the following lemma.

Lemma 3.2. Let $E$ and $F$ be two vector spaces and $\varphi$ and $\psi$ be two linear maps from $E$ to $F$ then $\operatorname{Ker} \varphi=\operatorname{Ker} \psi$ if and only if for all linear subspace $G$ of $E$, $\operatorname{dim} \varphi(G)=\operatorname{dim} \psi(G)$.

\section{Existence of equilibrium}

\subsection{Financial exchange economy}

We now consider a financial exchange economy, which is defined as the couple of an exchange economy $\mathcal{E}$ and a financial structure $\mathcal{F}$, which are linked by the portfolio sets of the consumers, which represent the sets of admissibles portfolios for each agent.

The stochastic exchange economy is described by a finite set of agent $\mathcal{I}$. Each agent $i \in \mathcal{I}$ has a consumption set $X_{i} \subset \mathbb{R}^{\mathbb{L}}$, which consists of all possible consumptions. An allocation is an element $x \in \prod_{i \in \mathcal{I}} X_{i}$ and we denote by $x_{i}$ the consumption of agent $i$, which is the projection of $x$ on $X_{i}$. 
The tastes of each consumer $i \in \mathcal{I}$ are represented by a strict preference correspondence $P_{i}: \prod_{k \in \mathcal{I}} X_{k} \longrightarrow X_{i}$, where $P_{i}(x)$ defines the set of consumptions that are strictly preferred to $x_{i}$ for agent $i$, given the consumption $x_{k}$ for the other consumers $k \neq i$. $P_{i}$ represents the consumer tastes, but also his behavior with respect to time and uncertainty, especially his impatience and attitude toward risk. If consumer preferences are represented by utility functions $u_{i}: X_{i} \longrightarrow \mathbb{R}$ for each $i \in \mathcal{I}$, the strict preference correspondence is defined by $P_{i}(x)=\left\{x_{i}^{\prime} \in X_{i} \mid u_{i}\left(x_{i}^{\prime}\right)>u_{i}\left(x_{i}\right)\right\}$.

For each node $\xi \in \mathbb{D}$, every consumer $i \in \mathcal{I}$ has a node endowment $e_{i}(\xi) \in \mathbb{R}^{\mathbb{H}}$ (contingent on the fact that $\xi$ prevails) and we denote by $e_{i}=\left(e_{i}(\xi)\right)_{\xi \in \mathbb{D}} \in \mathbb{R}^{\mathbb{L}}$ the endowments for the whole set of nodes. The exchange economy $\mathcal{E}$ can be summarized by

$$
\mathcal{E}=\left[\mathbb{D}, \mathbb{H}, \mathcal{I},\left(X_{i}, P_{i}, e_{i}\right)_{i \in \mathcal{I}}\right]
$$

We assume that each consumer $i$ is endowed with a portfolio set $Z_{i} \subset \mathbb{R}^{\mathcal{J}}$. For a discussion on this concept we refer to Angeloni-Cornet [1], Aouani-Cornet [2] and the references therein. The financial exchange economy can thus be summarized by

$$
(\mathcal{E}, \mathcal{F}):=\left[\mathbb{D}, \mathbb{H}, \mathcal{I},\left(X_{i}, P_{i}, e_{i}\right)_{i \in \mathcal{I}}, \mathcal{J},\left(Z_{i}\right)_{i \in \mathcal{I}},(\xi(j))_{j \in \mathcal{J}}, V\right]
$$

\subsection{Financial equilibrium and arbitrage}

Given the price $(p, q) \in \mathbb{R}^{\mathbb{L}} \times \mathbb{R}^{\mathcal{J}}$, the budget set of consumer $i \in \mathcal{I}$ is $B_{\mathcal{F}}^{i}(p, q)$ defined by ${ }^{5}$ :

$$
\left\{\left(x_{i}, z_{i}\right) \in X_{i} \times Z_{i}: \forall \xi \in \mathbb{D}, p(\xi) \bullet \mathbb{H}\left[x_{i}(\xi)-e_{i}(\xi)\right] \leq\left[W(p, q) z_{i}\right](\xi)\right\}
$$

or

$$
\left\{\left(x_{i}, z_{i}\right) \in X_{i} \times Z_{i}: p_{\square}\left(x_{i}-e_{i}\right) \leq W(p, q) z_{i}\right\}
$$

We now introduce the equilibrium notion:

Definition 4.1. An equilibrium of the financial exchange economy $(\mathcal{E}, \mathcal{F})$ is a list of strategies and prices $(\bar{x}, \bar{z}, \bar{p}, \bar{q}) \in\left(\mathbb{R}^{\mathbb{L}}\right)^{\mathcal{I}} \times\left(\mathbb{R}^{\mathcal{J}}\right)^{\mathcal{I}} \times \mathbb{R}^{\mathbb{L}} \backslash\{0\} \times \mathbb{R}^{\mathcal{J}}$ such that

(a) for every $i \in \mathcal{I},\left(\bar{x}_{i}, \bar{z}_{i}\right)$ maximizes the preferences $P_{i}$ in the budget set $B_{\mathcal{F}}(\bar{p}, \bar{q})$, in the sense that

$$
\left(\bar{x}_{i}, \bar{z}_{i}\right) \in B_{\mathcal{F}}^{i}(\bar{p}, \bar{q}) \text { and }\left[P_{i}(\bar{x}) \times Z_{i}\right] \bigcap B_{\mathcal{F}}^{i}(\bar{p}, \bar{q})=\emptyset
$$

(b) $\sum_{i \in \mathcal{I}} \bar{x}_{i}=\sum_{i \in \mathcal{I}} e_{i}$ (Commodity market clearing condition);

(c) $\sum_{i \in \mathcal{I}} \bar{z}_{i}=0$ (Portfolio market clearing condition).

\footnotetext{
${ }^{5}$ For $x=(x(\xi))_{\xi \in \mathbb{D}}, p=(p(\xi))_{\xi \in \mathbb{D}}$ in $\mathbb{R}^{\mathbb{L}}=\mathbb{R}^{\mathbb{H} \times \mathbb{D}}$ (with $x(\xi), p(\xi)$ in $\mathbb{R}^{\mathbb{H}}$ ) we let $p \square x=$ $\left(p(\xi) \bullet_{\mathbb{H}} x(\xi)\right)_{\xi \in \mathbb{D}} \in \mathbb{R}^{\mathbb{D}}$.
} 
Angeloni-Cornet[1] noted that when portfolios may be constrained, the concept of arbitrage free has to be suitably modified. In particular, we shall make a distinction between the definitions of arbitrage free portfolio and arbitrage free financial structure.

Definition 4.2. Given the financial structure $\mathcal{F}=\left(\mathcal{J},(\xi(j))_{j \in \mathcal{J}}, V\right)$, and the portfolio sets $\left(Z_{i}\right)_{i \in \mathcal{I}}$, the portfolio $\bar{z}_{i} \in Z_{i}$ is said with no arbitrage opportunities or to be arbitrage free for agent $i \in \mathcal{I}$ at the price $(p, q) \in \mathbb{R}^{\mathbb{L}} \times \mathbb{R}^{\mathcal{J}}$ if there is no portfolio $z_{i} \in Z_{i}$ such that $W(p, q) z_{i}>W(p, q) \bar{z}_{i}$, that is, $\left[W(p, q) z_{i}\right](\xi) \geq\left[W(p, q) \bar{z}_{i}\right](\xi)$, for every $\xi \in \mathbb{D}$, with at least one strict inequality, or, equivalently, if:

$$
W(p, q)\left(Z_{i}-\bar{z}_{i}\right) \cap \mathbb{R}_{+}^{\mathbb{D}}=\{0\} .
$$

The financial structure is said to be arbitrage free at $(p, q)$ if there exists no portfolio $\left(z_{i}\right) \in \prod_{i \in \mathcal{I}} Z_{i}$ such that $W(p, q)\left(\sum_{i \in \mathcal{I}} z_{i}\right)>0$, or, equivalently, if:

$$
W(p, q)\left(\sum_{i \in \mathcal{I}} Z_{i}\right) \cap \mathbb{R}_{+}^{\mathbb{D}}=\{0\} .
$$

Let the financial structure $\mathcal{F}$ be arbitrage free at $(p, q)$, and let $\left(\bar{z}_{i}\right) \in \prod_{i \in \mathcal{I}} Z_{i}$ such that $\sum_{i \in \mathcal{I}} \bar{z}_{i}=0$. Then, for every $i \in \mathcal{I}, \bar{z}_{i}$ is arbitrage free at $(p, q)$. The converse is true, for example, when some agent's portfolio set is unconstrained, that is, when $Z_{i}=\mathbb{R}^{\mathcal{J}}$ for some $i \in \mathcal{I}$.

The following characterization of arbitrage free portfolio is taken from Angeloni-Cornet [1].

Proposition 4.1. Let $\mathcal{F}=\left(\mathcal{J},(\xi(j))_{j \in \mathcal{J}}, V\right)$ and the portfolio set $\left(Z_{i}\right)_{i \in \mathcal{I}}$, let $(p, q) \in \mathbb{R}^{\mathbb{L}} \times \mathbb{R}^{\mathcal{J}}$, for $i \in \mathcal{I}$, let $z_{i} \in Z_{i}$, assume that $Z_{i}$ is convex and consider the following statements:

(i) There exists $\lambda_{i} \in \mathbb{R}_{++}^{\mathbb{D}}$ such that ${ }^{t} W(p, q) \lambda_{i} \in N_{Z_{i}}\left(z_{i}\right)^{6}$, or, equivalently, there exists $\eta \in N_{Z_{i}}\left(z_{i}\right)$ such that for every $j \in \mathcal{J}$,

$$
\lambda_{i \xi(j)} q_{j}=\sum_{\xi>\xi(j)} \lambda_{i \xi} V_{\xi}^{j}(p)-\eta_{j} .
$$

(ii) The portfolio $z_{i}$ is arbitrage free for agent $i \in \mathcal{I}$ at price $(p, q)$.

The implication $[(i) \Rightarrow(i i)]$ always holds and the converse is true under the additional assumption that $Z_{i}$ is a polyhedral ${ }^{7}$ set.

We recall that equilibrium portfolios are arbitrage free under the following non-satiation assumption:

\section{Assumption NS}

\footnotetext{
${ }^{6}$ we recall that $N_{Z_{i}}\left(z_{i}\right)$ is the normal cone to $Z_{i}$ at $z_{i}$, which is defined as $N_{Z_{i}}\left(z_{i}\right)=$ $\left\{\eta \in \mathbb{R}^{\mathcal{J}}: \eta \bullet \mathcal{J} z_{i} \geq \eta \bullet \mathcal{J} z_{i}^{\prime}, \forall z_{i}^{\prime} \in Z_{i}\right\}$

${ }^{7}$ A subset $C \subset \mathbb{R}^{n}$ is said to be polyhedral if it is the intersection of finitely many closed half-spaces, namely $C=\left\{x \in \mathbb{R}^{n}: A x \leq b\right\}$, where $A$ is a real $(m \times n)$-matrix , and $b \in \mathbb{R}^{m}$. Note that polyhedral sets are always closed and convex and that the empty set and the whole space $\mathbb{R}^{n}$ are both polyhedral.
} 
(i) (Non-Saturation at Every Node.) For every $\bar{x} \in \prod_{i \in \mathcal{I}} X_{i}$ if $\sum_{i \in \mathcal{I}} \bar{x}_{i}=$ $\sum_{i \in \mathcal{I}} e_{i}$, then, for every $i \in \mathcal{I}$, for every $\xi \in \mathbb{D}$, there exists $x_{i} \in X_{i}$ such that, for each $\xi^{\prime} \neq \xi, x_{i}\left(\xi^{\prime}\right)=\bar{x}_{i}\left(\xi^{\prime}\right)$ and $x_{i} \in P_{i}(\bar{x})$;

(ii) if $x_{i} \in P_{i}(\bar{x})$, then $\left.] \bar{x}_{i}, x_{i}\right] \subset P_{i}(\bar{x})$.

Proposition 4.2. Under Assumption (NS), if $(\bar{x}, \bar{z}, \bar{p}, \bar{q})$ is an equilibrium of the economy $(\mathcal{E}, \mathcal{F})$, then $\bar{z}_{i}$ is arbitrage free at price $(\bar{p}, \bar{q})$ for every $i \in \mathcal{I}$.

The proof is given in Angeloni-Cornet [1].

\subsection{The existence result}

From now on, we only consider nominal asset financial structure for which $V$ does not depend on the spot price $p$.

We introduce the following assumptions on the consumers and the financial structure. They are borrowed from Angeloni-Cornet [1] and Cornet-Gopalan [3]. In the following $\mathcal{Z}_{\mathcal{F}}$ is the linear space spanned by $\cup_{i \in \mathcal{I}} Z_{i}$.

Assumption C (Consumption Side) For all $i \in \mathcal{I}$ and all $\bar{x} \in \prod_{i \in \mathcal{I}} X_{i}$,

(i) $X_{i}$ is a closed, convex and bounded below subset of $\mathbb{R}^{\mathbb{L}}$;

(ii) the preference correspondence $P_{i}$, from $\prod_{k \in \mathcal{I}} X_{k}$ to $X_{i}$, is lower semicontinuous and $P_{i}(\bar{x})$ is convex;

(iii) for every $x_{i} \in P_{i}(\bar{x})$ for every $x_{i}^{\prime} \in X_{i}, x_{i}^{\prime} \neq x_{i},\left[x_{i}^{\prime}, x_{i}\left[\cap P_{i}(\bar{x}) \neq \emptyset^{8}\right.\right.$;

(iv) (Irreflexivity) $\bar{x}_{i} \notin P_{i}(\bar{x})$;

(v) (Non-Saturation of Preferences at Every Node) if $\sum_{i \in \mathcal{I}} \bar{x}_{i}=\sum_{i \in \mathcal{I}} e_{i}$, for every $i \in \mathcal{I}$, for every $\xi \in \mathbb{D}$, there exists $x_{i} \in X_{i}$ such that, for each $\xi^{\prime} \neq \xi, x_{i}\left(\xi^{\prime}\right)=\bar{x}_{i}\left(\xi^{\prime}\right)$ and $x_{i} \in P_{i}(\bar{x})$;

(vi) (Strong Survival Assumption) $e_{i} \in \operatorname{int} X_{i}$.

Note that these assumptions on $P_{i}$ are satisfied when agents' preferences are represented by a continuous, strongly monotone and quasi-concave utility function.

\section{Assumption F (Financial Side)}

(i) for every $i \in \mathcal{I}, Z_{i}$ is a closed, convex subset of $\mathbb{R}^{\mathcal{J}}$ containing 0 ;

(ii) there exists $i_{0} \in \mathcal{I}$ such that $0 \in \operatorname{ri}_{\mathcal{Z}_{\mathcal{F}}}\left(Z_{i_{0}}\right)^{9}$.

\footnotetext{
${ }^{8}$ This is satisfied, in particular, when $P_{i}(\bar{x})$ is open in $X_{i}$ (for its relative topology ).

${ }^{9}$ Let $Z$ a nonempty subset of $\mathbb{R}^{\mathcal{J}}$ and let $H$ a subspace of $\mathbb{R}^{\mathcal{J}}$ such that $Z \subset H$. We call relative interior of $Z$ with respect to $H$ denoted $\operatorname{ri}_{H}(Z)$ the set $\left\{z \in \mathbb{R}^{\mathcal{J}} \mid \exists r>0 ; B(z, r) \cap H \subset\right.$ $Z\}$.
} 
Note that we slightly weaken the assumption of Angeloni-Cornet [1] since we consider the linear space $\mathcal{Z}_{\mathcal{F}}$ instead of $\mathbb{R}^{\mathcal{J}}$ for the relative interior. Nevertheless, Assumption $\mathrm{F}$ is stronger than the corresponding one in Cornet-Gopalan [3] (Assumption FA), which is that the closed cone spanned by $\cup_{i \in \mathcal{I}} W(q)\left(Z_{i}\right)$ is a linear space. Indeed, if $0 \in \operatorname{ri}_{\mathcal{Z}_{\mathcal{F}}}\left(Z_{i_{0}}\right)$, then the cone spanned by $W(q)\left(Z_{i_{0}}\right)$ is equal to $W(q)\left(\mathcal{Z}_{\mathcal{F}}\right)$, which is a linear space and since $Z_{i} \subset \mathcal{Z}_{\mathcal{F}}$ for all $i$, $W(q)\left(Z_{i}\right) \subset W(q)\left(\mathcal{Z}_{\mathcal{F}}\right)$. Hence, the cone spanned by $\cup_{i \in \mathcal{I}} W(q)\left(Z_{i}\right)$ is equal to $W(q)\left(\mathcal{Z}_{\mathcal{F}}\right)$, which is a linear space.

Our main existence result is the following:

Proposition 4.3. Let

$$
(\mathcal{E}, \mathcal{F}):=\left[\mathbb{D}, \mathbb{H}, \mathcal{I},\left(X_{i}, P_{i}, e_{i}\right)_{i \in \mathcal{I}}, \mathcal{J},\left(Z_{i}\right)_{i \in \mathcal{I}},(\xi(j))_{j \in \mathcal{J}}, V\right]
$$

be a financial economy with nominal assets satisfying Assumptions $\mathbf{C}, \mathbf{R}, \mathbf{F}$ and such that $\operatorname{Ker} V \cap \mathcal{Z}_{\mathcal{F}}=\{0\}$. Then, for any given $\lambda \in \mathbb{R}_{++}^{\mathbb{D}}$, there exists a financial equilibrium $(\bar{x}, \bar{z}, \bar{p}, \bar{q})$ where $\bar{q}$ satisfies

$$
{ }^{t} W(\bar{q}) \lambda \in N_{Z_{i_{0}}}\left(\bar{z}_{i_{0}}\right)
$$

Our contribution is to obtain an existence result with long-term assets with assumptions only on the fundamentals of the economy, namely the payoff matrix $V$ and the portfolio sets $Z_{i}$, regardless of the arbitrage free price. Note that in Cornet-Gopalan [3], Assumption FA depends on the asset price $q$, which is an endogenous variable.

Remark 4.1. $\mathcal{Z}_{\mathcal{F}} \cap \operatorname{Ker} V=\{0\}$ is a slight weakening of the usual assumption of no redundant assets. To remove this assumption, as in [2] in a two-period model, we need to consider an auxiliary economy with a projection of the portfolio sets, which is a topic of further research.

The proof of our existence result is based upon Theorem 3.1 of AngeloniCornet [1]. To state this theorem, we need to introduce the set $B^{\delta}(\lambda)$ of $\delta$ admissible consumptions and portfolios for a given state price $\lambda \in \mathbb{R}_{++}^{\mathbb{D}}$, that is, the set of consumption-portfolio pair $(x, z) \in \prod_{i \in \mathcal{I}} X_{i} \times \prod_{i \in \mathcal{I}} Z_{i}$ such that there exists a commodity-asset price pair $(p, q) \in \bar{B}_{\mathbb{L}}(0,1) \times \mathbb{R}^{\mathcal{J}}$ satisfying:

$$
\left\{\begin{array}{l}
{ }^{t} W(p, q) \lambda \in \bar{B}_{\mathcal{J}}(0, \delta), \\
\left(x_{i}, z_{i}\right) \in B_{\mathcal{F}}^{i}(p, q) \forall i \in \mathcal{I}, \\
\sum_{i \in \mathcal{I}} x_{i}=\sum_{i \in \mathcal{I}} e_{i}, \\
\sum_{i \in \mathcal{I}} z_{i}=0
\end{array}\right.
$$

The standard existence result requires that the set $B^{1}(\lambda)$ is bounded but the proof can be easily adapted to the case where $B^{\delta}(\lambda)$ is bounded for some $\delta>0$ (see the proof of Proposition 4.3 below). In [1], it is proved that this holds true if the assets are all short-term and $\operatorname{rank} V=\# \mathcal{J}$ or, if there are long-term assets, that $\operatorname{rank} W(p, q)=\# \mathcal{J}$ for all $(p, q, \eta) \in B_{L}(0,1) \times \mathbb{R}^{\mathcal{J}} \times B_{J}(0,1)$ such that ${ }^{t} W(p, q) \lambda=\eta$. Note that $B^{1}(\lambda)$ may be not bounded under the assumptions of Proposition 4.3 but Assumption R.

Indeed, let a financial structure with the same tree $\mathbb{D}$ as in Remark 3.1 above. At each non-terminal node, two assets are issued, hence $J=6$. The return matrix $V$ is constant and equal to 


$$
\mathbf{V}=\left[\begin{array}{llllll}
0 & 0 & 0 & 0 & 0 & 0 \\
1 & 2 & 0 & 0 & 0 & 0 \\
1 & 1 & 0 & 0 & 0 & 0 \\
2 & 1 & 1 & 0 & 0 & 0 \\
1 & 1 & 1 & 1 & 0 & 0 \\
1 & 1 & 0 & 0 & 1 & 0 \\
1 & 1 & 0 & 0 & 0 & 1
\end{array}\right] \begin{aligned}
& \xi_{0} \\
& \xi_{1} \\
& \xi_{2} \\
& \xi_{11} \\
& \xi_{21} \\
& \xi_{22}
\end{aligned}
$$

One remarks that the rank of the matrix $V$ is 6 . So the kernel of $V$ is reduced to $\{0\}$ hence $\operatorname{Ker} V \cap \mathcal{Z}_{\mathcal{F}}=\{0\}$ whatever is the linear space $\mathcal{Z}_{\mathcal{F}}$. We now consider the asset price $q=(7,7,2,1,1,1) . q$ is an arbitrage free price since ${ }^{t} W(q) \lambda=0$ with $\lambda=(1,1,1,1,1,1,1) \in \mathbb{R}_{++}^{7}$. Hence the full-return matrix is

$$
\mathbf{W}(\mathbf{q})=\left[\begin{array}{cccccc}
-7 & -7 & 0 & 0 & 0 & 0 \\
1 & 2 & -2 & -1 & 0 & 0 \\
1 & 1 & 0 & 0 & -1 & -1 \\
2 & 1 & 1 & 0 & 0 & 0 \\
1 & 1 & 1 & 1 & 0 & 0 \\
1 & 1 & 0 & 0 & 1 & 0 \\
1 & 1 & 0 & 0 & 0 & 1
\end{array}\right] \begin{aligned}
& \xi_{0} \\
& \xi_{1} \\
& \xi_{11} \\
& \xi_{12} \\
& \xi_{21} \\
& \xi_{22}
\end{aligned}
$$

The rank of $W(q)$ is 5 since the dimension of the kernel of $W(q)$ is 1 .

Let us consider

$$
(\mathcal{E}, \mathcal{F}):=\left[\mathbb{D}, \mathbb{H}, \mathcal{I},\left(X_{i}, P_{i}, e_{i}\right)_{i \in \mathcal{I}}, \mathcal{J},\left(Z_{i}\right)_{i \in \mathcal{I}},(\xi(j))_{j \in \mathcal{J}}, V\right]
$$

a financial exchange economy satisfying Assumption $\mathrm{C}$ with $X_{i}=\mathbb{R}_{+}^{7}, \mathcal{I}=2$, $\mathbb{H}$ is a singleton and $Z_{i}=\mathbb{R}^{6}$.

Let $\left(z_{i}^{\nu}\right)$ be a sequence of elements of $\mathbb{R}^{6}$ such that

$$
z_{1}^{\nu}=-z_{2}^{\nu}=\nu(1,-1,-1,1,0,0)
$$

The spot price is $p=(1,1,1,1,1,1,1)$. Let $\hat{x}_{1}=e_{1}=(3,3,3,3,3,3,3)=\hat{x}_{2}=$ $e_{2}$. For all $\nu,\left(\hat{x}, z^{\nu}\right) \in B^{1}(\lambda)$ since for all $\nu \in \mathbb{N},{ }^{t}\left[W(q) z_{1}^{\nu}\right]=0={ }^{t}\left[W(q) z_{2}^{\nu}\right]$. Hence $B^{1}(\lambda)$ is not bounded since $\left(z^{\nu}\right)$ is not bounded.

The proof of Proposition 4.3 is divided into two steps. We first prove that the set $B^{\delta}(\lambda)$ is bounded for $\delta>0$ small enough (Proposition 4.4). Then, we deduce the existence of an equilibrium under this additional assumption from Theorem 3.1 of Angeloni-Cornet [1] with a slight adaptation of the proof to deal with the space $\mathcal{Z}_{\mathcal{F}}$ instead of $\mathbb{R}^{\mathcal{J}}$ and $B^{\delta}(\lambda)$ instead of $B^{1}(\lambda)$.

Proposition 4.4. Let

$$
(\mathcal{E}, \mathcal{F}):=\left[\mathbb{D}, \mathbb{H}, \mathcal{I},\left(X_{i}, P_{i}, e_{i}\right)_{i \in \mathcal{I}}, \mathcal{J},\left(Z_{i}\right)_{i \in \mathcal{I}},(\xi(j))_{j \in \mathcal{J}}, V\right]
$$

be a financial economy satisfying for all $i \in \mathcal{I}, X_{i}$ is bounded below, $\mathcal{F}$ consists of nominal assets and satisfies Assumption $(\mathbf{F})$. Let $\lambda \in \mathbb{R}_{++}^{\mathbb{D}}$ and $q$ be the unique asset price such that ${ }^{t} W(q) \lambda=0$. If $\mathcal{Z}_{\mathcal{F}} \cap \operatorname{Ker} W(q)=\{0\}$, there exists $\delta>0$ such that $B^{\delta}(\lambda)$ is bounded. 


\section{Appendix}

Proof of Proposition 3.1 Let $p \in \mathbb{R}^{\mathbb{L}}$ be a spot price vector. Let $\bar{q} \in \mathbb{R}^{\mathcal{J}}$ an asset price and let $\left(q^{\nu}\right)$ a sequence of $Q_{p}$, which converges to $\bar{q} \in Q_{p}$ and let $\bar{w} \in H(p, \bar{q})$. Let $\bar{z} \in \mathbb{R}^{\mathcal{J}}$ such that $\bar{w}=W(p, \bar{q}) \bar{z}$. Then the sequence $\left(w^{\nu}=W\left(p, q^{\nu}\right) \bar{z}\right)$ converges to $\bar{w}$ since the sequence of matrices $\left(W\left(p, q^{\nu}\right)\right)$ converges to $W(p, \bar{q})$ and $w^{\nu} \in H\left(p, q^{\nu}\right)$ from the very definition of $H(p, \cdot)$. Hence the correspondance $H(p,$.$) is l.s.c. on Q_{p}$.

Proof of Proposition 3.2 Let $\left(q^{\nu}\right)$ a sequence of $Q_{p}$ converging to $\bar{q} \in Q_{p}$ and let $\left(w^{\nu}\right)$ a sequence of marketable payoffs converging to $\bar{w}$ such that, for each $\nu$, $w^{\nu} \in H\left(p, q^{\nu}\right)$. We prove that $\bar{w} \in H(p, \bar{q})$.

For each $\nu$ there exists $z^{\nu} \in \mathbb{R}^{\mathcal{J}}$ such that $w^{\nu}=W\left(p, q^{\nu}\right) z^{\nu}$. Let $\hat{z}^{\nu}$ be the orthogonal projection of $z^{\nu}$ on $(\operatorname{Ker} V(p))^{\perp}$. Then $w^{\nu}=W\left(p, q^{\nu}\right) \hat{z}^{\nu}+W\left(p, q^{\nu}\right)\left(z^{\nu}-\right.$ $\left.\hat{z}^{\nu}\right)=W\left(p, q^{\nu}\right) \hat{z}^{\nu}$ since $z^{\nu}-\hat{z}^{\nu} \in \operatorname{Ker} V(p)=\operatorname{Ker} W\left(p, q^{\nu}\right)$ by assumption.

We now prove that the sequence $\left(\hat{z}^{\nu}\right)$ is bounded. Indeed, suppose, by contradiction, that this is not true. Then, there exists a subsequence $\left(\hat{z}^{\phi(\nu)}\right)$ such that $\left\|\hat{z}^{\phi(\nu)}\right\| \rightarrow+\infty$. For each $\nu$, let $\zeta^{\nu}=\frac{\hat{z}^{\phi(\nu)}}{\left\|\hat{z}^{\phi(\nu)}\right\|}$

The sequence $\left(\zeta^{\nu}\right)$ belongs to the unit sphere. So there exists a subsequence $\left(\zeta^{\psi(\nu)}\right)$ of $\left(\zeta^{\nu}\right)$ which converges to $\bar{\zeta}$. Clearly $\|\bar{\zeta}\|=1$ and and $\bar{\zeta} \in(\operatorname{Ker} V(p))^{\perp}$ since $\hat{z}^{\nu} \in(\operatorname{Ker} V(p))^{\perp}$ for all $\nu$. Thus, for each $\nu$, we have

$$
W\left(p, q^{\nu}\right) \frac{\hat{z}^{\phi \circ \psi(\nu)}}{\left\|\hat{z}^{\phi \circ \psi(\nu)}\right\|}=W\left(p, q^{\nu}\right) \zeta^{\psi(\nu)} \rightarrow W(p, \bar{q}) \bar{\zeta}
$$

and

$$
W\left(p, q^{\nu}\right) \zeta^{\psi(\nu)}=W\left(p, q^{\nu}\right) \frac{\hat{z}^{\phi \circ \psi(\nu)}}{\left\|\hat{z}^{\phi \circ \psi(\nu)}\right\|}=\frac{w^{\phi \circ \psi(\nu)}}{\left\|\hat{z}^{\phi \circ \psi(\nu)}\right\|} \rightarrow 0
$$

since $\left(w^{\nu}\right)$ is bounded and $\left\|\hat{z}^{\phi(\nu)}\right\| \rightarrow+\infty$. Thus $\bar{\zeta} \in \operatorname{Ker} W(p, \bar{q})=\operatorname{Ker} V(p)$ by assumption and $\bar{\zeta} \in(\operatorname{Ker} V(p))^{\perp}$, so $\bar{\zeta}=0$ which contradicts $\|\bar{\zeta}\|=1$.

Since the sequence $\left(\hat{z}^{\nu}\right)$ is bounded, there exists a converging subsequence $\left(\hat{z}^{\varphi(\nu)}\right)$ which converges to $\bar{z} \in(\operatorname{Ker} V)^{\perp}$ and we easily checks that

$$
\bar{w}=\lim _{\nu \rightarrow+\infty} w^{\varphi(\nu)}=\lim _{\nu \rightarrow+\infty} W\left(p, q^{\varphi(\nu)}\right) z^{\varphi(\nu)}=W(p, \bar{q}) \bar{z} \in H(p, \bar{q}) .
$$

Proof of Proposition 3.4. Let $q$ be an arbitrage free price and let $\lambda=\left(\lambda_{\xi}\right) \in$ $\mathbb{R}_{++}^{\mathbb{D}}$ such that ${ }^{t} W(p, q) \lambda=0$. From Proposition 3.5, $\operatorname{rank} V(p)=\operatorname{rank} W(p, q)$ and this implies that $\operatorname{dim} \operatorname{Ker} V(p)=\operatorname{dim} \operatorname{Ker} W(p, q)$. So, to get the equality of the kernels, it remains to show $\operatorname{Ker} V(p) \subset \operatorname{Ker} W(p, q)$.

Let $z=\left(z^{j}\right)_{j \in \mathcal{J}} \in \mathbb{R}^{\mathcal{J}}$ be an element of the kernel of the payoff matrice $V(p)$, so $\sum_{j \in \mathcal{J}} z^{j} V^{j}(p)=0$. From Proposition 3.5, $\operatorname{Im} V(p)=\bigoplus_{\xi \in \mathbb{D}^{e}} \operatorname{Im} V^{\mathcal{J}(\xi)}(p)$, hence $\sum_{j \in \mathcal{J}(\xi)} z^{j} V^{j}(p)=0$ for all $\xi \in \mathbb{D}^{e}$. 
For all $\xi \in \mathbb{D}^{e}$, for all $j \in \mathcal{J}(\xi)$ and for all $\eta \in \mathbb{D} \backslash\{\xi\}, V_{\eta}^{j}(p)=W_{\eta}^{j}(p, q)$. At the node $\xi, \sum_{j \in \mathcal{J}(\xi)} z^{j} W_{\xi}^{j}(p, q)=-\sum_{j \in \mathcal{J}(\xi)} z^{j} q_{j}$. But

$$
q_{j}=\left(1 / \lambda_{\xi}\right) \sum_{\xi^{\prime} \in \mathbb{D}^{+}(\xi)} \lambda_{\xi^{\prime}} V_{\xi^{\prime}}^{j}(p) .
$$

Hence,

$$
\begin{aligned}
\sum_{j \in \mathcal{J}(\xi)} z^{j} q_{j} & =\left(1 / \lambda_{\xi}\right) \sum_{j \in \mathcal{J}(\xi)} z^{j}\left[\sum_{\xi^{\prime} \in \mathbb{D}^{+}(\xi)} \lambda_{\xi^{\prime}} V_{\xi^{\prime}}^{j}(p)\right] \\
& =\left(1 / \lambda_{\xi}\right) \sum_{\xi^{\prime} \in \mathbb{D}^{+}(\xi)} \lambda_{\xi^{\prime}}\left[\sum_{j \in \mathcal{J}(\xi)} z^{j} V_{\xi^{\prime}}^{j}(p)\right] \\
& =0
\end{aligned}
$$

So, we have proved that $\sum_{j \in \mathcal{J}(\xi)} z^{j} W^{j}(p, q)=0$, and since it holds true for all $\xi \in \mathbb{D}^{e}, \sum_{j \in \mathcal{J}} z^{j} W^{j}(p, q)=0$ that is $z \in \operatorname{Ker} W(p, q)$.

Proof of Proposition 3.5. For all $\xi \in \mathbb{D}^{e}$, we denote by $n(\xi)$ the number of assets issued at this node and by $\operatorname{rk}(\xi)$ the rank of $V_{\mathbb{D}}^{\mathcal{J}(\xi)}(p)$. We also simplify the notation by defining $V^{\mathcal{J}(\xi)}(p):=V_{\mathbb{D}}^{\mathcal{J}(\xi)}(p)$ and $W^{\mathcal{J}(\xi)}(p, q):=W_{\mathbb{D}}^{\mathcal{J}(\xi)}(p, q)$.

Step 1: For all $\xi \in \mathbb{D}^{e}, \operatorname{rank} W^{\mathcal{J}(\xi)}(p, q)=\operatorname{rk}(\xi)$.

Let us consider $\lambda=\left(\lambda_{\xi}\right)_{\xi \in \mathbb{D}} \in \mathbb{R}_{++}^{\mathbb{D}}$ such that ${ }^{t} W(p, q) \lambda=0$. Such $\lambda$ exists since $q$ is an arbitrage free price.

For all $\xi \in \mathbb{D}^{e}$, let $\mathcal{J}^{\prime}(\xi) \subset \mathcal{J}(\xi)$ such that $\# \mathcal{J}^{\prime}(\xi)=\operatorname{rk}(\xi)$ and the family $\left(V_{\xi}^{j}(p)\right)_{j \in \mathcal{J}^{\prime}(\xi)}$ is linearly independent. Since $W^{\mathcal{J}^{\prime}(\xi)}(p, q)$ is obtained from $V^{\mathcal{J}^{\prime}(\xi)}(p)$ by replacing a zero row by the row of the opposite of asset prices issued at $\xi$, the regular $\operatorname{rk}(\xi)$ square sub-matrix of $V^{\mathcal{J}^{\prime}(\xi)}(p)$ is also a sub-matrix of $W^{\mathcal{J}^{\prime}(\xi)}(p, q)$, hence the rank of $W^{\mathcal{J}^{\prime}(\xi)}(p, q)$ is higher or equal to $\operatorname{rk}(\xi)^{10}$.

Let us now prove that the rank of $W^{\mathcal{J}(\xi)}(p, q)$ is not strictly larger than $\operatorname{rk}(\xi)$. It suffices to prove that for all $j_{0} \notin \mathcal{J}^{\prime}(\xi), W^{j_{0}}(p, q) \in \operatorname{Vect}\left(\left(W^{j}(p, q)\right)_{j \in \mathcal{J}^{\prime}(\xi)}\right)$.

$V^{j_{0}}(p)$ is a linear combination of $\left(V^{j}(p)\right)_{j \in \mathcal{J}^{\prime}(\xi)}$ since the rank of $V^{\mathcal{J}(\xi)}(p)$ is $\operatorname{rk}(\xi)$. Hence there exists $\left(\alpha_{j}\right)_{j \in \mathcal{J}^{\prime}(\xi)}$ such that $\sum_{j \in \mathcal{J}^{\prime}(\xi)} \alpha_{j} V^{j}(p)=V^{j_{0}}(p)$. Since ${ }^{t} W(p, q) \lambda=0, \lambda_{\xi} q_{j_{0}}=\sum_{\xi^{\prime} \in \mathbb{D}^{+}(\xi)} \lambda_{\xi^{\prime}} V_{\xi^{\prime}}^{j_{0}}(p)$. Hence $\lambda_{\xi} q_{j_{0}}$ is equal to

$$
\begin{aligned}
\sum_{\xi^{\prime} \in \mathbb{D}^{+}(\xi)}\left[\lambda_{\xi^{\prime}} \sum_{j \in \mathcal{J}^{\prime}(\xi)} \alpha_{j} V_{\xi^{\prime}}^{j}(p)\right] & =\sum_{j \in \mathcal{J}^{\prime}(\xi)}\left[\alpha_{j} \sum_{\xi^{\prime} \in \mathbb{D}^{+}(\xi)} \lambda_{\xi^{\prime}} V_{\xi^{\prime}}^{j}(p)\right] \\
& =\sum_{j \in \mathcal{J}^{\prime}(\xi)}\left[\alpha_{j} \lambda_{\xi} q_{j}\right]=\lambda_{\xi} \sum_{j \in \mathcal{J}^{\prime}(\xi)} \alpha_{j} q_{j}
\end{aligned}
$$

Hence $q_{j_{0}}=\sum_{j \in \mathcal{J}^{\prime}(\xi)} \alpha_{j} q_{j}$. Since $\sum_{j \in \mathcal{J}^{\prime}(\xi)} \alpha_{j} V^{j}(p)=V^{j_{0}}(p)$, we obtain for all $\eta \neq \xi$

$$
\sum_{j \in \mathcal{J}^{\prime}(\xi)} \alpha_{j} W_{\eta}^{j}(p, q)=\sum_{j \in \mathcal{J}^{\prime}(\xi)} \alpha_{j} V_{\eta}^{j}(p)=V_{\eta}^{j_{0}}(p)=W_{\eta}^{j_{0}}(p, q)
$$

and

$$
\sum_{j \in \mathcal{J}^{\prime}(\xi)} \alpha_{j} W_{\xi}^{j}(p, q)=-\sum_{j \in \mathcal{J}^{\prime}(\xi)} \alpha_{j} q_{j}=-q_{j_{0}}=W_{\xi}^{j_{0}}(p, q)
$$

\footnotetext{
${ }^{10}$ Note that we do not use the fact that the asset price is an arbitrage free price in this part of the proof.
} 
So,

$$
\sum_{j \in \mathcal{J}^{\prime}(\xi)} \alpha_{j} W^{j}(p, q)=W^{j_{0}}(p, q)
$$

and $W^{j_{0}}(p, q)$ belongs to $\left.\operatorname{Vect}\left(W^{j}(p, q)\right)_{j \in \mathcal{J}^{\prime}(\xi)}\right)$.

Step 2. $\operatorname{rank} V(p)=\sum_{\xi \in \mathbb{D}^{e}} \operatorname{rk}(\xi)=\operatorname{rank} W(p, q), \operatorname{Im} V(p)=\bigoplus_{\xi \in \mathbb{D}^{e}} \operatorname{Im} V^{\mathcal{J}(\xi)}(p)$ and $\operatorname{Im} W(p, q)=\bigoplus_{\xi \in \mathbb{D}^{e}} \operatorname{Im} W^{\mathcal{J}(\xi)}(p, q)$.

We first remark that $\operatorname{Vect}(V(p))=+_{\xi \in \mathbb{D}^{e}} \operatorname{Vect}\left(V^{\mathcal{J}(\xi)}(p)\right)$ which implies using Step 2 that $\operatorname{rank} V(p) \leq \sum_{\xi \in \mathbb{D}^{e}} \operatorname{rank} V^{\mathcal{J}(\xi)}(p)=\sum_{\xi \mathbb{D}^{e}} \operatorname{rk}(\xi)$.

Like in the proof of Step 1, for all $\xi \in \mathbb{D}^{e}$, let $\mathcal{J}^{\prime}(\xi) \subset \mathcal{J}(\xi)$ such that $\# \mathcal{J}^{\prime}(\xi)=\operatorname{rk}(\xi)$ and the family $\left(V_{\xi}^{j}(p)\right)_{j \in \mathcal{J}^{\prime}(\xi)}$ is linearly independent. Let $\mathcal{J}^{\prime}=$ $\cup_{\xi \in \mathbb{D}^{e}} \mathcal{J}^{\prime}(\xi)$ and for all $\kappa=1, \ldots, k, \mathcal{J}_{\kappa}^{\prime}=\cup_{\xi \in \mathbb{D}_{\tau_{\kappa}}^{e}} \mathcal{J}^{\prime}(\xi)$. We now prove that the family $\left(V^{j}(p)\right)_{j \in \mathcal{J}^{\prime}}$ is linearly independent. Note that this implies $\operatorname{rank} V(p)=$ $\sum_{\xi \in \mathbb{D}^{e}} \operatorname{rk}(\xi)$ and $\operatorname{Im} V(p)=\bigoplus_{\xi \in \mathbb{D}^{e}} \operatorname{Im} V^{\mathcal{J}(\xi)}(p)$ since $\left(V^{j}(p)\right)_{j \in \mathcal{J}^{\prime}(\xi)}$ is a basis of $\operatorname{Im} V^{\mathcal{J}(\xi)}(p)$ for all $\xi$.

Let $\left(\alpha_{j}\right) \in \mathbb{R}^{\mathcal{J}^{\prime}}$ such that $\sum_{j \in \mathcal{J}^{\prime}} \alpha_{j} V^{j}(p)=0$. We work by backward induction on $\kappa$ from $k$ to 1 .

For all $\xi \in \mathbb{D}_{\tau_{k}}^{e}, \sum_{j \in \mathcal{J}^{\prime}} \alpha_{j} V_{\mathbb{D}^{+}(\xi)}^{j}(p)=0$. Since $\tau_{\kappa}<\tau_{k}$ for all $\kappa=1, \ldots, k-1$, for all $j$ such that $\xi(j) \notin \mathbb{D}^{-}(\xi) \cup\{\xi\}, V_{\mathbb{D}^{+}(\xi)}^{j}(p)=0$. So, one gets

$$
\sum_{j \in \mathcal{J}^{\prime}(\xi)} \alpha_{j} V_{\mathbb{D}^{+}(\xi)}^{j}(p)+\sum_{\xi^{\prime} \in \mathbb{D}^{-}(\xi)} \sum_{j \in \mathcal{J}^{\prime}\left(\xi^{\prime}\right)} \alpha_{j} V_{\mathbb{D}^{+}(\xi)}^{j}(p)=0
$$

From Assumption R,

$$
\operatorname{Vect}\left(V_{\mathbb{D}^{+}(\xi)}^{\mathcal{J}\left(\mathbb{D}^{-}(\xi)\right)}(p)\right) \bigcap \operatorname{Vect}\left(V_{\mathbb{D}^{+}(\xi)}^{\mathcal{J}(\xi)}(p)\right)=\{0\} .
$$

From the above equality,

$$
\sum_{j \in \mathcal{J}^{\prime}(\xi)} \alpha_{j} V_{\mathbb{D}^{+}(\xi)}^{j}(p) \in \operatorname{Vect}\left(V_{\mathbb{D}^{+}(\xi)}^{\mathcal{J}\left(\mathbb{D}^{-}(\xi)\right)}(p)\right) \bigcap \operatorname{Vect}\left(V_{\mathbb{D}^{+}(\xi)}^{\mathcal{J}(\xi)}(p)\right)
$$

hence $\sum_{j \in \mathcal{J}^{\prime}(\xi)} \alpha_{j} V_{\mathbb{D}^{+}(\xi)}^{j}(p)=0$.

By construction, the family $\left(V^{j}(p)\right)_{j \in \mathcal{J}^{\prime}(\xi)}$ is linearly independent and for all $\xi^{\prime} \notin \mathbb{D}^{+}(\xi), V_{\xi^{\prime}}^{j}(p)=0$, so the family $\left(V_{\mathbb{D}^{+}(\xi)}^{j}(p)\right)_{j \in \mathcal{J}^{\prime}(\xi)}$ is linearly independent. Hence, from above, one deduces that $\alpha_{j}=0$ for all $j \in \mathcal{J}^{\prime}(\xi)$. Since this is true for all $\xi \in \mathbb{D}_{\tau_{k}}^{e}$, one gets $\alpha_{j}=0$ for all $j \in \mathcal{J}_{k}^{\prime}$.

If $k=1$, we are done. If $k \geq 2$, we do again the same argument as above. Indeed, since we have proved that for all $j \in \mathcal{J}_{k}^{\prime}, \alpha_{j}=0$, for all $\xi \in \mathbb{D}_{\tau_{k-1}}^{e}$, $\sum_{j \in \mathcal{J}^{\prime}} \alpha_{j} V_{\mathbb{D}^{+}(\xi)}^{j}(p)=0$ implies $\sum_{j \in \mathcal{J}^{\prime} \backslash \mathcal{J}_{k}^{\prime}} V_{\mathbb{D}^{+}(\xi)}^{j}(p)=0$, hence

$$
\sum_{j \in \mathcal{J}^{\prime}(\xi)} \alpha_{j} V_{\mathbb{D}^{+}(\xi)}^{j}(p)+\sum_{\xi^{\prime} \in \mathbb{D}^{-}(\xi)} \sum_{j \in \mathcal{J}^{\prime}\left(\xi^{\prime}\right)} \alpha_{j} V_{\mathbb{D}^{+}(\xi)}^{j}(p)=0 .
$$


Using again Assumption $\mathbf{R}$ and the linear independence of $\left(V_{\mathbb{D}^{+}(\xi)}^{j}(p)\right)_{j \in \mathcal{J}^{\prime}(\xi)}$, one then deduces that for all $j \in \mathcal{J}_{k-1}^{\prime}, \alpha_{j}=0$.

Consequently, after a finite number of steps, we deduce that all $\alpha_{j}$ are equal to 0 , which implies that the family $\left(V^{j}(p)\right)_{j \in \mathcal{J}^{\prime}}$ is linearly independent.

For the remaining result about the matrix $W(p, q)$, we prove that the family $\left(W^{j}(p, q)\right)_{j \in \mathcal{J}^{\prime}}$ is linearly independent. We use a similar argument noticing that for all $j \in \mathcal{J}^{\prime}(\xi) \cup\left(\cup_{\xi^{\prime} \in \mathbb{D}^{-}(\xi)} J^{\prime}\left(\xi^{\prime}\right)\right), V_{\mathbb{D}^{+}(\xi)}^{j}(p)=W_{\mathbb{D}^{+}(\xi)}^{j}(p, q)$, which implies that for all $\xi \in \mathbb{D}^{e}$, the family $\left(W^{j}(p, q)\right)_{j \in \mathcal{J}^{\prime}(\xi)}$ is linearly independent.

Proof of Proposition 3.6. 1) The proof is just an adaptation of the proof of Proposition 3.5. In the first step, since the price $q$ is not supposed to be a arbitrage free price, we get $\operatorname{rank} W^{\mathcal{J}(\xi)}(p, q) \geq \operatorname{rk}(\xi)$ instead of an equality. For the second step, the proofs never uses the fact that $q$ is an arbitrage free price, so we can replicate them to obtain $\operatorname{rank} W(p, q) \geq \sum_{\xi \in \mathbb{D}^{e}} \operatorname{rk}(\xi)=\operatorname{rank} V(p)$.

2) If $\operatorname{rk}(\xi)=n(\xi)$ for all $\xi$, then $\sum_{\xi \in \mathbb{D}^{e}} \operatorname{rk}(\xi)$ is the cardinal of $\mathcal{J}$, which is the number of column of the matrix $W(p, q)$. So $\operatorname{rank} W(p, q) \leq \sum_{\xi \in \mathbb{D}^{e}} \operatorname{rk}(\xi)=$ $\operatorname{rank} V(p)$.

Proof of Corollary 3.1 First, we remark that $V_{\xi^{+}}^{\mathcal{J}(\xi)}(p)$ is a sub-matrix of $V^{\mathcal{J}(\xi)}(p)$, so $n(\xi)=\operatorname{rank} V_{\xi^{+}}^{\mathcal{J}(\xi)}(p) \leq \operatorname{rank} V^{\mathcal{J}(\xi)}(p)$. On the other hand, $\operatorname{rank} V^{\mathcal{J}(\xi)}(p) \leq n(\xi)$ since the number of column of $V^{\mathcal{J}(\xi)}(p)$ is $n(\xi)$. Hence $n(\xi)=\operatorname{rank} V^{\mathcal{J}(\xi)}(p)$.

We now prove that Assumption $\mathbf{R}$ is satisfied. Let $\kappa \in\{2, \ldots, k\}, \xi \in \mathbb{D}_{\tau_{\kappa}}^{e}$ and $y \in \mathbb{R}^{\mathbb{D}^{+}(\xi)} \backslash\{0\}$ such that

$$
y \in \operatorname{Vect}\left(V_{\mathbb{D}^{+}(\xi)}^{\mathcal{J}\left(\mathbb{D}^{-}(\xi)\right)}(p)\right) \cap \operatorname{Vect}\left(V_{\mathbb{D}^{+}(\xi)}^{\mathcal{J}(\xi)}(p)\right)
$$

Then, there exists $\left(a_{j}\right) \in \mathbb{R}^{\mathcal{J}(\xi)}$ such that $y=\sum_{j \in \mathcal{J}(\xi)} a_{j} V_{\mathbb{D}^{+}(\xi)}^{j}(p)$ and there exists $\left(b_{j}\right) \in \mathbb{R}^{\mathcal{J}\left(\mathbb{D}^{-}(\xi)\right)}$ such that $y=\sum_{j \in \mathcal{J}\left(\mathbb{D}^{-}(\xi)\right)} b_{j} V_{\mathbb{D}^{+}(\xi)}^{j}(p)$. Restricting the above equality to the coordinates in $\xi^{+}$, one gets $y_{\xi^{+}}=\sum_{j \in \mathcal{J}(\xi)} a_{j} V_{\xi^{+}}^{j}(p)=$ $\sum_{j \in \mathcal{J}\left(\mathbb{D}^{-}(\xi)\right)} b_{j} V_{\xi^{+}}^{j}(p)$. From our second assumption, this implies that $y_{\xi^{+}}=$ 0 . From the first assumption, since the vectors $\left(V_{\xi^{+}}^{j}(p)\right)_{j \in \mathcal{J}(\xi)}$ are of maximal rank hence linearly independent, this implies that $a_{j}=0$ for all $j \in \mathcal{J}(\xi)$. Hence, $y=0$, which proves that $\operatorname{Vect}\left(V_{\mathbb{D}^{+}(\xi)}^{\mathcal{J}\left(\mathbb{D}^{-}(\xi)\right)}(p)\right) \cap \operatorname{Vect}\left(V_{\mathbb{D}^{+}(\xi)}^{\mathcal{J}(\xi)}(p)\right)=$ $\{0\}$. Consequently Assumption $\mathbf{R}$ is satisfied.

Proof of Corollary 3.2. We show Part A and with the same reasoning we can show Part B thanks to Assumption R. Obviously, (ii) implies (i). Let us show the other implication.

According to the rank Theorem and thanks to Proposition 3.5, we have $\operatorname{dim} \operatorname{Ker} V(p)=\# \mathcal{J}-\operatorname{dim} \operatorname{Im} V(p)=\sum_{\xi \in \mathbb{D}^{e}} \# \mathcal{J}(\xi)-\sum_{\xi \in \mathbb{D}^{e}} \operatorname{dim} \operatorname{Im} V^{\mathcal{J}(\xi)}(p)=$ $\sum_{\xi \in \mathbb{D}^{e}}\left[\# \mathcal{J}(\xi)-\operatorname{dim} \operatorname{Im} V^{\mathcal{J}(\xi)}(p)\right]=\sum_{\xi \in \mathbb{D}^{e}} \operatorname{dim} \operatorname{Ker} V^{\mathcal{J}(\xi)}(p)$. If, for every $\xi \in$ 
$\mathbb{D}^{e}$, the family $\left\{V^{j}(p)\right\}_{j \in \mathcal{J}(\xi)}$ is linearly independent, then, $\operatorname{dim} \operatorname{Ker} V^{\mathcal{J}(\xi)}(p)=$ 0 . From the previous equality, $\operatorname{dim} \operatorname{Ker} V(p)=0$, hence, the family $\left\{V^{j}(p)\right\}_{j \in \mathcal{J}}$ is linearly independent. So $(i)$ implies $(i i)$.

Proof of lemma 3.1. Let us denote by $k$ [resp. $\left.k^{\prime}\right]$ the number of dates where there are issuance of at least one asset for the financial structure $\mathcal{F}$ [resp. $\left.\mathcal{F}^{\prime}\right]$. It is clear that $k^{\prime} \leq k$.

By Assumption $\mathbf{R}$, we have: for all $\kappa \in\{2, \ldots, k\}$ and for all $\xi \in \mathbb{D}_{\tau_{\kappa}}^{e}$,

$$
\operatorname{Vect}\left(V_{\mathbb{D}^{+}(\xi)}^{\mathcal{J}\left(\mathbb{D}^{-}(\xi)\right.}\right) \bigcap \operatorname{Vect}\left(V_{\mathbb{D}^{+}(\xi)}^{\mathcal{J}(\xi)}\right)=\{0\}
$$

Since $\mathcal{J}^{\prime} \subset \mathcal{J}, \operatorname{Vect}\left(V_{\mathbb{D}^{+}(\xi)}^{\mathcal{J}^{\prime}\left(\left(\mathbb{D}^{-}(\xi)\right)\right.}\right) \subset \operatorname{Vect}\left(V_{\mathbb{D}^{+}(\xi)}^{\mathcal{J}\left(\left(\mathbb{D}^{-}(\xi)\right)\right.}\right)$ and $\operatorname{Vect}\left(V_{\mathbb{D}^{+}(\xi)}^{\mathcal{J}^{\prime}(\xi)}\right) \subset$ $\operatorname{Vect}\left(V_{\mathbb{D}+(\xi)}^{\mathcal{J}(\xi)}\right)$. So,

$$
\operatorname{Vect}\left(V_{\mathbb{D}^{+}(\xi)}^{\mathcal{J}^{\prime}\left(\xi^{-}\right)}\right) \cap \operatorname{Vect}\left(V_{\mathbb{D}^{+}(\xi)}^{\mathcal{J}^{\prime}(\xi)}\right) \subset \operatorname{Vect}\left(V_{\mathbb{D}^{+}(\xi)}^{\mathcal{J}\left(\mathbb{D}^{-}(\xi)\right.}\right) \bigcap \operatorname{Vect}\left(V_{\mathbb{D}^{+}(\xi)}^{\mathcal{J}(\xi)}\right)=\{0\} .
$$

hence the financial structure $\mathcal{F}^{\prime}$ satisfies Assumption $\mathbf{R}$.

Proof of lemma 3.2. We first show that the equality of the kernels implies the equality of dimensions of the images. Let $G$ be a linear subspace of $E$ and let $\varphi_{G}\left(\right.$ resp. $\left.\psi_{G}\right)$ be the restriction of $\varphi($ resp. $\psi)$ at $G$. We have $\varphi(G)=\operatorname{Im} \varphi_{G}$ and $\operatorname{dim} \operatorname{Im} \varphi_{G}=\operatorname{dim} G-\operatorname{dim}\left(\operatorname{Ker} \varphi_{G}\right) . \operatorname{As} \operatorname{Ker} \varphi=\operatorname{Ker} \psi$, we have $\operatorname{Ker} \varphi_{G}=$ $(\operatorname{Ker} \varphi) \cap G=(\operatorname{Ker} \psi) \cap G=\operatorname{Ker} \psi_{G}$ hence $\operatorname{dim} \varphi(G)=\operatorname{dim} \psi(G)$.

Let us show the converse implication. If $\operatorname{Ker} \varphi \neq \operatorname{Ker} \psi$, then there exists $u \in \operatorname{Ker} \varphi$ such that $u \notin \operatorname{Ker} \psi$ or there exists $u \in \operatorname{Ker} \psi$ such that $u \notin \operatorname{Ker} \varphi$. In the first case, with $G=\operatorname{Ker} \varphi$, we have $\varphi(G)=\{0\} \neq \psi(G)$, hence $\operatorname{dim} \varphi(G)=$ $0<\operatorname{dim} \psi(G)$. In the second case, we obtain the same inequality with $G=\operatorname{Ker} \psi$. So the equality of the dimension of $\varphi(G)$ and $\psi(G)$ for all linear subspace $G$ implies the equality of kernels.

Proof of Proposition 4.3 Let $\lambda \in \mathbb{R}_{++}^{\mathbb{D}}$ and $q$ be the unique asset price such that ${ }^{t} W(q) \lambda=0$. Since $\mathcal{Z}_{\mathcal{F}} \cap \operatorname{Ker} V=\{0\}$, Assumption $\mathbf{R}$ and Proposition 3.4 imply that $\mathcal{Z}_{\mathcal{F}} \cap \operatorname{Ker} W(q)=\{0\}$. So Proposition 4.4 implies that there exists $\delta>0$ such that $B^{\delta}(\lambda)$ is bounded. Hence, all assumptions of Theorem 3.1 of Angeloni-Cornet [1] are satisfied but the fact that $0 \in \operatorname{ri}_{\mathcal{Z}_{\mathcal{F}}}\left(Z_{i_{0}}\right)$ instead of $0 \in \operatorname{int} Z_{i_{0}}$ and $B^{\delta}(\lambda)$ is bounded instead of $B^{1}(\lambda)$. To complete the proof, we now show how to adapt the proof of Angeloni-Cornet to these slightly more general conditions.

In the preliminary definitions, $\eta$ is chosen in $\mathcal{Z}_{\mathcal{F}}$ instead of $\mathbb{R}^{\mathcal{J}}$. Then the set $B$ is replaced by

$$
B^{\delta}=\left\{(p, \eta) \in \mathbb{R}^{\mathbb{L}} \times \mathcal{Z}_{\mathcal{F}} \mid\|p\| \leq 1,\|\eta\| \leq \delta\right\}
$$

and the function $\rho$ is defined by $\rho(p, \eta)=\max \{0,1-\|p\|-(1 / \delta)\|\eta\|\}$. This choice of the set $B^{\delta}$ allows us to conclude in Sub-sub-section 4.1 .3 that $(\bar{x}, \bar{z}, \bar{p}, \bar{q})$ is 
an equilibrium and furthermore $(\bar{x}, \bar{z})$ belongs to $B^{\delta}(\lambda)$, which is used in the proof of Proposition 4.2 on page 22. In Step 2 of the proof of Claim 4.1, if $\eta \neq 0$, we obtain $0<\max \left\{\eta \bullet \mathcal{J} z_{i_{0}} \mid z_{i_{0}} \in Z_{i_{0}}\right\}$ since $Z_{i_{0}}$ is included in $\mathcal{Z}_{\mathcal{F}}$, $\eta \in \mathcal{Z}_{\mathcal{F}}$ and $0 \in \operatorname{ri}_{\mathcal{Z}_{\mathcal{F}}}\left(Z_{i_{0}}\right)$, so $r \eta \in Z_{i_{0}}$ for $r>0$ small enough. In Claim 4.3 of Sub-sub-section 4.1.3, the argument holds true since $\bar{z}_{i} \in \mathcal{Z}_{\mathcal{F}}$ for all $i$ and so, $\left(\delta /\left\|\sum_{i \in \mathcal{I}} \bar{z}_{i}\right\|\right) \sum_{i \in \mathcal{I}} \bar{z}_{i}$ belongs to $\mathcal{Z}_{\mathcal{F}}$. The equality $\sum_{i \in I}\left(\bar{x}_{i}-e_{i}\right)=0$ is obtained by the same argument. Indeed, since $\lambda_{\xi}>0$ for all $\xi, p \rightarrow(\lambda \square p) \bullet \mathbb{L}$ $\sum_{i \in I}\left(\bar{x}_{i}-e_{i}\right)$ is a non zero linear mapping if $\sum_{i \in I}\left(\bar{x}_{i}-e_{\underline{i}}\right) \neq 0$ so its maximum on the ball is positive and reached on the boundary of $\bar{B}_{\mathbb{L}}(0,1)$, which implies that $\|\bar{p}\|=1$ and $\rho(\bar{p}, \bar{\eta})=0$.

In Sub-sub-section 4.2 .2 , to show that $0 \in \operatorname{ri}_{\mathcal{Z}_{\mathcal{F}}}\left(Z_{i_{0} r}\right)$ in the truncated economy, it suffices to remark that there exists $r^{\prime}>0$ such that $B_{\mathcal{J}}\left(0, r^{\prime}\right) \cap \mathcal{Z}_{\mathcal{F}} \subset Z_{i_{0}}$, hence, $B_{\mathcal{J}}\left(0, \min \left\{r, r^{\prime}\right\}\right) \cap \mathcal{Z}_{\mathcal{F}} \subset Z_{i_{0} r}$, which means that 0 belongs to the relative interior of $Z_{i_{0} r}$ with respect to $\mathcal{Z}_{\mathcal{F}}$.

Proof of Proposition 4.4 For every $\delta>0$, for every $i \in \mathcal{I}, \lambda \in \mathbb{R}_{++}^{\mathbb{D}}$, we let $\hat{X}_{i}^{\delta}(\lambda)$ and $\hat{Z}_{i}^{\delta}(\lambda)$ be the projections of $B^{\delta}(\lambda)$ on $X_{i}$ and $Z_{i}$, that is respectively:

$$
\begin{aligned}
& \hat{X}_{i}^{\delta}(\lambda):=\left\{x_{i} \in X_{i} \mid \exists\left(x_{k}\right)_{k \neq i} \in \prod_{k \neq i} X_{k}, \exists z \in \prod_{k \in \mathcal{I}} Z_{k},(x, z) \in B^{\delta}(\lambda)\right\} \\
& \hat{Z}_{i}^{\delta}(\lambda):=\left\{z_{i} \in Z_{i} \mid \exists\left(z_{k}\right)_{k \neq i} \in \prod_{k \neq i} Z_{k}, \exists x \in \prod_{k \in \mathcal{I}} X_{k},(x, z) \in B^{\delta}(\lambda)\right\} .
\end{aligned}
$$

It suffices to prove that $\hat{X}_{i}^{\delta}(\lambda)$ and $\hat{Z}_{i}^{\delta}(\lambda)$ are bounded sets for every $i$ to show that $B^{\delta}(\lambda)$ is bounded

Step 1. Let us show that: for all $\delta \geq 0$, and for all $i \in \mathcal{I}, \hat{X}_{i}^{\delta}(\lambda)$ is bounded.

Indeed, let $\delta \geq 0$, and $i \in \mathcal{I}$, since the sets $X_{i}$ are bounded below, there exists $\underline{x}_{i} \in \mathbb{R}^{\mathbb{L}}$ such that $X_{i} \subset\left\{\underline{x}_{i}\right\}+\mathbb{R}_{+}^{\mathbb{L}}$. If $x_{i} \in \hat{X}_{i}(\lambda)$, there exists $x_{k} \in X_{k}$, for every $k \neq i$, such that $x_{i}+\sum_{k \neq i} x_{k}=\sum_{\kappa \in \mathcal{I}} e_{\kappa}$. Consequently,

$$
\underline{x}_{i} \leq x_{i}=-\sum_{k \neq i} x_{k}+\sum_{\kappa \in \mathcal{I}} e_{\kappa} \leq-\sum_{k \neq i} \underline{x}_{k}+\sum_{\kappa \in \mathcal{I}} e_{\kappa}
$$

and so $\hat{X}_{i}^{\delta}(\lambda)$ is bounded. So for all $\delta \geq 0$ and $i \in \mathcal{I}, \hat{X}_{i}^{\delta}(\lambda)$ is bounded.

Step 2. Let us show that for all $i \in \mathcal{I}, \hat{Z}_{i}^{0}(\lambda)$ is bounded.

For all $i \in \mathcal{I}$ and for every $z_{i} \in \hat{Z}_{i}^{0}(\lambda)$, there exists $\left(z_{k}\right)_{k \neq i} \in \prod_{k \neq i} Z_{k}$, $x \in \prod_{\kappa \in \mathcal{I}} X_{\kappa}$ and $p \in \bar{B}_{\mathbb{L}}(0,1)$, such that $z_{i}+\sum_{k \neq i} z_{k}=0$ and $\left(x_{\kappa}, z_{\kappa}\right) \in$ $B_{\mathcal{F}}^{\kappa}(p, q)$ for every $\kappa \in \mathcal{I}$. As $\left(x_{\kappa}, z_{\kappa}\right) \in B_{\mathcal{F}}^{\kappa}(p, q)$ and $\left(x_{\kappa}, p\right) \in \hat{X}_{j}^{0}(\lambda) \times \bar{B}_{\mathbb{L}}(0,1)$, a compact set, there exists $\alpha_{j} \in \mathbb{R}^{\mathbb{D}}$ such that

$$
\alpha_{j} \leq p_{\square}\left(x_{\kappa}-e_{\kappa}\right) \leq W(q) z_{\kappa} .
$$

Using the fact that $\sum_{\kappa \in \mathcal{I}} z_{\kappa}=0$, we have

$$
\alpha_{i} \leq W(q) z_{i}=W(q)\left(-\sum_{k \neq i} z_{k}\right) \leq-\sum_{k \neq i} \alpha_{k},
$$


hence there exists $r>0$ such that $W(q) z_{i} \in \bar{B}_{\mathbb{D}}(0, r)$.

By assumption, $\mathcal{Z}_{\mathcal{F}} \cap \operatorname{Ker} W(q)=\{0\}$. So, the linear mapping $W(q)_{\mid \mathcal{Z}_{\mathcal{F}}}$ from $\mathcal{Z}_{\mathcal{F}}$ to $W(q) \mathcal{Z}_{\mathcal{F}}$ is an isomorphism. Since we have proved that for every $z_{i} \in \hat{Z}_{i}^{0}(\lambda), W(q) z_{i} \in \bar{B}_{\mathbb{D}}(0, r)$ and $W(q) z_{i}$ obviously belongs to $W(q) \mathcal{Z}_{\mathcal{F}}$, we can conclude that $\hat{Z}_{i}^{0}(\lambda) \subset\left[W(q)_{\mid \mathcal{Z}_{\mathcal{F}}}\right]^{-1}\left(\bar{B}_{\mathbb{D}}(0, r) \cap W(q) \mathcal{Z}_{\mathcal{F}}\right)$, a bounded subset, so $\hat{Z}_{i}^{0}(\lambda)$ is a bounded subset of $\mathcal{Z}_{\mathcal{F}}$.

Let $M \in \mathbb{R}_{+}^{*}$ such that for all $(x, z) \in B^{0}(\lambda),\|z\|<M$.

Step 3. There exists $\delta>0$ such that $B^{\delta}(\lambda)$ is bounded.

By contradiction. Suppose that for all $\delta>0, B^{\delta}(\lambda)$ is not bounded. This implies that for all $\nu \in \mathbb{N}^{*}, B^{1 / \nu}(\lambda)$ is not bounded. We build a sequence $\left(x^{\nu}, z^{\nu}\right)_{\nu \in \mathbb{N}^{*}}$ in $\prod_{i \in \mathcal{I}} X_{i} \times \prod_{i \in \mathcal{I}} Z_{i}$ by induction in the following way: $\left(x^{1}, z^{1}\right) \in$ $B^{1}(\lambda)$ such that $\left\|z^{1}\right\|>M+1$ and for all $\nu \in \mathbb{N}^{*},\left(x^{\nu+1}, z^{\nu+1}\right) \in B^{\frac{1}{\nu+1}}(\lambda)$ and $\left\|z^{\nu+1}\right\|>\left\|z^{\nu}\right\|+1$. So $\left\|z^{\nu}\right\|$ converges to $+\infty$.

Since for all $\nu \in \mathbb{N}^{*},\left(x^{\nu}, z^{\nu}\right) \in B^{1 / \nu}(\lambda)$, there exists a sequence $\left(p^{\nu}, q^{\nu}\right)_{\nu \in \mathbb{N}^{*}}$ such that for all $\nu \in \mathbb{N}^{*},\left\|p^{\nu}\right\| \leq 1, p^{\nu}$ 口 $\left(x_{i}^{\nu}-e_{i}\right) \leq W\left(q^{\nu}\right) z_{i}^{\nu}$ and $0 \leq\left\|{ }^{t} W\left(q^{\nu}\right) \lambda\right\| \leq$ $\frac{1}{\nu}$. We remark that for all $\nu \in \mathbb{N}^{*}, B^{\frac{1}{\nu+1}}(\lambda) \subset B^{\frac{1}{\nu}}(\lambda)$ so the sequence $\left(x^{\nu}, z^{\nu}\right) \subset$ $B^{1}(\lambda)$. By Step 1 , the sequence $\left(x^{\nu}\right)$ is bounded. For each $\nu \in \mathbb{N}^{*}$, let $\zeta^{\nu}=$ $M \frac{z^{\nu}}{\left\|z^{\nu}\right\|} \cdot{ }^{t} W\left(q^{\nu}\right) \lambda \in \bar{B}_{\mathcal{J}}\left(0, \frac{1}{\nu}\right)$ implies that for all $\nu \in \mathbb{N}^{*}$ and for all $j \in \mathcal{J}$ there exists $\eta^{\nu} \in \bar{B}_{\mathcal{J}}\left(0, \frac{1}{\nu}\right)$ such that $\lambda_{\xi(j)} q^{\nu j}=\sum_{\xi \in \mathbb{D}^{+}(\xi(j))} \lambda_{\xi} V_{\xi}^{j}+\eta^{\nu j}$. Hence the sequence $\left(q^{\nu j}\right)$ is bounded for all $j$. Consequently the sequence $\left(x^{\nu}, \zeta^{\nu}, p^{\nu}, q^{\nu}\right)$ is bounded so it has a subsequence $\left(x^{\phi(\nu)}, \zeta^{\phi(\nu)}, p^{\phi(\nu)}, q^{\phi(\nu)}\right)$, which converges to $(\bar{x}, \bar{\zeta}, \bar{p}, \bar{q})$.

Let us now show that $(\bar{x}, \bar{\zeta}) \in B^{0}(\lambda)$.

- ${ }^{t} W(\bar{q}) \lambda=0$ since $\left\|{ }^{t} W\left(q^{\phi(\nu)}\right) \lambda\right\| \leq \frac{1}{\phi(\nu)}$ for all $\nu$. For all $i, \bar{x}_{i} \in X_{i}$ because $X_{i}$ is a closed. For all $i, \bar{\zeta}_{i} \in Z_{i}$. Indeed, $Z_{i}$ is closed and $\zeta_{i}^{\phi(\nu)}=M \frac{z_{i}^{\phi(\nu)}}{\left\|z^{\phi(\nu)}\right\|} \in Z_{i}$ since $z_{i}^{\phi(\nu)} \in Z_{i}, 0 \in Z_{i}, 0<\frac{M}{\left\|z^{\phi(\nu)}\right\|}<1$ and $Z_{i}$ is convex.

- For all $i,\left(\bar{x}_{i}, \bar{\zeta}_{i}\right) \in B_{\mathcal{F}}^{i}(0, \bar{q})$. Indeed, for all $\nu \in \mathbb{N}^{*},\left(x^{\nu}, z^{\nu}\right) \in B^{1}(\lambda)$, hence $p^{\phi(\nu)} \square\left(x_{i}^{\phi(\nu)}-e_{i}\right) \leq W\left(q^{\phi(\nu)}\right) z_{i}^{\phi(\nu)}$. So $\left(\frac{M}{\left\|z^{\phi(\nu)}\right\|^{\phi(\nu)}}\right) \square\left(x_{i}^{\phi(\nu)}-e_{i}\right) \leq$ $W\left(q^{\phi(\nu)}\right)\left(\frac{M}{\left\|z^{\phi(\nu)}\right\|} z_{i}^{\phi(\nu)}\right)$. At the limit, since $\zeta_{i}^{\phi(\nu)}=\frac{M z^{\phi(\nu)}}{\left\|z^{\phi(\nu)}\right\|}$ and $\frac{M}{\left\|z^{\phi(\nu)}\right\|}$ converges to 0 , one gets, $0 \leq W(\bar{q}) \bar{\zeta}_{i}$, which means that $\left(\bar{x}_{i}, \bar{\zeta}_{i}\right) \in B_{\mathcal{F}}^{i}(0, \bar{q})$.

- $\sum_{i \in \mathcal{I}} \bar{x}_{i}=\sum_{i \in \mathcal{I}} e_{i}$ and $\sum_{i \in \mathcal{I}} \bar{\zeta}_{i}=0$ since for all $\nu, \sum_{i \in \mathcal{I}} x_{i}^{\phi(\nu)}=\sum_{i \in \mathcal{I}} e_{i}$ and $\sum_{i \in \mathcal{I}} \zeta_{i}^{\phi(\nu)}=\sum_{i \in \mathcal{I}} M \frac{z_{i}^{\phi(\nu)}}{\left\|z^{\phi(\nu)}\right\|}=0$.

Hence, one gets a contradiction since $(\bar{x}, \bar{\zeta}) \in B^{0}(\lambda)$ and $\|\bar{\zeta}\|=M$ whereas we have chosen $M$ large enough so that for all $(x, z) \in B^{0}(\lambda),\|z\|<M$. 


\section{Acknowledgments}

We would like to thank Bernard Cornet for very helpful comments, Zaier Aouani, who has detected a mistake after a careful reading of a previous version of the paper and an anonymous referee for her/his fruitful suggestion to explicitly state the marketable payoff set as a direct sum of the marketable payoff sets associated to each issuance node.

\section{References}

[1] L. Angeloni and B. Cornet. Existence of financial equilibria in a multi-period stochastic economy. Mathematical Economics, 8:1-31, 2006.

[2] Z. Aouani and B. Cornet. Existence of financial equilibria with restricted participations. Journal of Mathematical Economics, 45:772-786, 2009.

[3] B. Cornet and R. Gopalan. Arbitrage and equilibrium with portfolio constraints. Economic Theory, 45:227-252, 2010.

[4] B. Cornet and A. Ranjan. A remark on arbitrage free prices in multi-period economy. Working paper, University of Paris 1, 2012.

[5] M. Magill and M. Quinzii. Theory of Incomplete Markets. Cambridge, 1996. 